\title{
Screening for functional circular RNAs using the CRISPR-Cas13 system
}

Siqi $\mathrm{Li}^{1,4}$, Xiang $\mathrm{Li}^{1,4}$, Wei Xue ${ }^{2,4}$, Lin Zhang ${ }^{1,4}$, Shi-Meng $\mathrm{Cao}^{1}$, Yun-Ni Lei ${ }^{2,3}$, LiangZhong Yang ${ }^{1}$, Si-Kun Guo ${ }^{1}$, Jia-Lin Zhang ${ }^{2}$, Xiang $\mathrm{Gao}^{1,3}$, Jia $\mathrm{Wei}^{2}$, Jinsong $\mathrm{Li}^{1,3 *}$, Li Yang $^{2,3, *}$, Ling-Ling Chen ${ }^{1,3, *}$

${ }^{1}$ State Key Laboratory of Molecular Biology, Shanghai Key Laboratory of Molecular Andrology, CAS Center for Excellence in Molecular Cell Science, Shanghai Institute of Biochemistry and Cell Biology, University of Chinese Academy of Sciences, Chinese Academy of Sciences, 320 Yueyang Road, Shanghai 200031, China

${ }^{2}$ CAS Key Laboratory of Computational Biology, CAS-MPG Partner Institute for Computational Biology, Shanghai Institute of Nutrition and Health, Shanghai Institutes for Biological Sciences, University of Chinese Academy of Sciences, Chinese Academy of Sciences, 320 Yueyang Road, Shanghai 200031, China

${ }^{3}$ School of Life Science and Technology, ShanghaiTech University, 100 Haike Road, Shanghai 201210, China

${ }^{4}$ These authors contributed equally

* Correspondence should be addressed to L.Y. (liyang@picb.ac.cn), J. L. (jsli@sibcb.ac.cn) and L.-L.C. (linglingchen@sibcb.ac.cn) 


\section{Summary}

Circular RNAs (circRNAs) produced from back-spliced exons are widely expressed, but individual circRNA functions remain poorly understood due to inadequate methods, such as RNAi and genome engineering, in distinguishing overlapped exons in circRNAs from those in linear cognate mRNAs ${ }^{1,2}$. Here we report that the programable RNA-guided, RNA-targeting CRISPR-Cas13, RfxCas13d, effectively and specifically discriminates circRNAs from mRNAs, using guide (g)RNAs targeting sequences spanning the back-splicing junction (BSJ) sites featured in RNA circles. Using a lentiviral library that targets sequences across BSJ sites of highly expressed human circRNAs, we show that a group of circRNAs are important for cell growth mostly in a cell-type specific manner and that a common oncogenic circRNA, circFAM120A, promotes cell proliferation in vitro and in vivo by preventing FAM120A mRNA from binding the translation inhibitor IGF2BP2 for efficient translation. Application of RfxCas $13 \mathrm{~d} / \mathrm{BSJ}$-gRNA screening has also uncovered circManla2 with regulatory potential in mouse embryo preimplantation development. Together, these results establish CRISPR-RfxCas13d as a useful tool for the discovery and functional study of circRNAs at both individual and large-scale levels.

\section{Introduction}

Back-splicing (BS) of pre-mRNA exons leads to genome-wide expression of circRNAs that are identical to linear mRNA sequences except at the BS junction (BSJ) sites. Their functions largely remain elusive, partly owing to the lack of effective tools to interfere with circular but not linear RNA levels in cells ${ }^{1}$. Short hairpin RNAs (shRNAs) or specific small interfering RNAs (siRNAs) targeting BSJ sites of circRNAs have been used to knock down circRNAs ${ }^{3-5}$. However, even partial complementarity of a halfRNAi ( $\sim 10 \mathrm{nt})$ to linear mRNAs may affect parental gene expression (Extended Data Fig. 1a). Genome engineering has been used to deplete a circRNA by removing either the entire circRNA-forming exon ${ }^{6}$ or the intronic cis-elements required for circRNA biogenesis $^{7,8}$ (Extended Data Fig. 1b), but only a few circRNAs were studied with these approaches ${ }^{6-8}$. A simple and scalable tool that can be used to identify functional circRNAs is still lacking.

Recent studies have established that RNA-targeting type VI CRISPR effectors, known as Cas13a, Cas13b and Cas13d RNases ${ }^{9-12}$, can be directed to cleave single-stranded (ss) RNA targets carrying complementary protospacers by a CRISPR RNA in mammalian cells ${ }^{11-14}$. Type VI CRISPR-Cas knockdown requires 22- to 30-nt spacers (guide RNAs, gRNAs), exhibits higher specificity than RNAi, and is intolerant to mismatches in the central seed region that hybridizes with target RNAs ${ }^{11-14}$. However, whether the CRISPR-Cas 13 system can discriminate circRNAs from their linear cognates with gRNAs targeting sequences spanning the BSJ, and if yes, the robustness and broad application of this approach on circRNA levels have remained unknown.

\section{RfxCas13d/BSJ-gRNA discriminates circRNAs from mRNAs}

We first sought to identify the best effector for circRNA knockdown by screening Cas 13 family proteins ${ }^{11-15}$. We synthesized the mammalian codon-optimized DNA sequence for each protein (LwaCas13a, PspCas13b, PguCas13b, RanCas13b, EsCas13d, AdmCas13d or RfxCas13d) and cloned them individually into an expression vector 
with a sequence for C-terminal monomeric superfolder GFP (msfGFP) to enhance protein stability (Extended Data Fig. 1c). Each Cas13 was expressed in 293FT cells (Extended Data Fig. 1d-e) and various knockdown efficiencies of KARS mRNA were observed when Cas13 and matched gRNAs were co-transfected into cells (Extended Data Fig. 1f), showing that these Cas 13 proteins are capable of targeted RNA cleavage using specific gRNAs.

Next, we examined the knockdown efficiency and specificity of each Cas 13 on two circRNAs, $\operatorname{circPOLR} 2 A$ (336 nt) and $\operatorname{circRTN4}(2,457 \mathrm{nt})$. We designed three gRNAs spanning BSJ (BSJ-gRNAs) for each circRNA (Fig. 1a). Most Cas13s (except LwaCas 13a) could attenuate expression of both circRNAs but had no detectable effect on their linear cognate mRNAs (Fig. 1b; Extended Data Fig. 1g), suggesting that the Cas 13 system could discriminate circRNAs from paired linear mRNAs by BSJ-gRNAs. Among all examined Cas 13 constructs, RfxCas13d exhibited the highest knockdown efficiency $(>80 \%)$ for both circRNAs, while linear POLR2A and RTN4 mRNAs remained largely unchanged (Fig. 1b; Extended Data Fig. 1g). Northern Blot analysis further confirmed the dramatic knockdown of $\operatorname{circPOLR2A}$ by RfxCas13d/BSJ-gRNAs (Extended Data Fig. 1h). Importantly, a non-targeting (NT) gRNA did not direct Cas 13 to circRNAs for cleavage (Fig. 1b; Extended Data Fig. 1g); overexpression of RfxCas13d or individual BSJ-gRNAs alone did not affect circRNA expression (Extended Data Fig. 1i-j). To rule out off-target effects of BSJ-gRNAs, we designed additional control gRNAs having half-sequences replaced by scrambled sequences (paired control) (Fig. 1c) or sequences from adjacent linear exons (gRNA-L) (Extended Data Fig. 1k) of circPOLR2A and $\operatorname{circRTN4}$, respectively. None of these control gRNAs could direct RfxCas13d to degrade corresponding circRNAs (Fig. 1c and Extended Data Fig. 1k). As expected, however, control gRNAs (gRNA-L) targeting linear RNA exon-exon junctions led to decreased linear mRNA expression by RfxCas $13 \mathrm{~d}$ (Extended Data Fig. 1k).

To explore the general applicability of RfxCas13d/BSJ-gRNAs for circRNA interference, we examined the knockdown efficiencies of another eight abundant circRNAs in human cells (Fig. 1d). Similar to $\operatorname{circPOLR2A}$ and $\operatorname{circRTN4}$, expression of all examined circRNAs, but not paired linear mRNAs, decreased more than $50 \%$ when RfxCas13d and BSJ-gRNAs vectors were co-transfected into 293FT cells (Fig. 1d). In HeLa cells, we found that RfxCas13d was the most efficient Cas13 to specifically disrupt expression of circRNAs (Extended Data Fig. 2a-c).

siRNAs $s^{5,16,17}$ and shRNAs ${ }^{3,4}$ were used in loss-of-function (LOF) studies of circRNAs. We compared the specificity and knockdown efficiency of the RfxCas $13 \mathrm{~d}$ system and shRNAs in a position-matched manner for nine pairs of circular and linear cognate RNAs (Fig. 1e, top). Compared to AGO2/shRNA-mediated knockdown, RfxCas13d/BSJ-gRNA showed much higher efficiency of circRNA knockdown (RfxCas13d: mean 70\%; shRNA: mean 34\%) and much lower off-target rates on linear mRNAs for all examined pairs (Fig. 1e, bottom). Collectively, these results reveal that RfxCas $13 \mathrm{~d}$ is an effective tool for circRNA knockdown with high efficiency, specificity and generality.

\section{Characteristics of RfxCas13d/BSJ-gRNA-mediated circRNA knockdown}

We characterized key features required for RfxCas13d/BSJ-gRNA-mediated circRNA knockdown. We first determined the minimal spacer length requirement for an efficient 
RfxCas13d targeting for circRNAs. We generated a series of BSJ-gRNAs with spacers ranging from 16 36 nt, symmetrically spanning the BSJ of circPOLR2A (Fig. 1f, top). Consistent with the previous in vitro cleavage assay of EsCas $13 \mathrm{~d}^{11}$, a minimal spacer of $18 \mathrm{nt}$ was required for RfxCas13d to target $\operatorname{circPOLR} 2 A$, but the highest knockdown efficiency was observed with $22 \mathrm{nt}$ or longer spacers (Fig. 1f, bottom left). In all examined conditions, we observed that the expression of linear POLR2A mRNA was barely affected (Fig. 1f, bottom right), suggesting that recognizing the unique BSJ in $\operatorname{circPOLR} 2 A$ (considered as a mismatch ribonucleotide for the paired linear mRNA) in the spacer could effectively constrain RfxCas13d targeting to only the circRNA.

This notion was further confirmed by characterizing the mismatch tolerance of RfxCas13d for circRNA targeting. We introduced either single or double mismatches into BSJ-gRNAs and observed that any single- or double-mismatch strikingly impaired the knockdown efficiency of $\operatorname{circPOLR} 2 A$ by RfxCas13d (Fig. 1g). A central seed region of the BSJ-gRNA spacer was identified as being more sensitive to mismatches, extending from the position of -8 to $8 \mathrm{nt}$ in a $22 \mathrm{nt}$ long BSJ-gRNA spacer (Fig. 1g). As expected, expression of linear $P O L R 2 A$ mRNA remained unaltered under all conditions (Fig. 1g).

We evaluated the knockdown efficiency and specificity of RfxCas $13 \mathrm{~d}$ for circRNA and its paired linear mRNA by designing gRNAs tiled in 10-nt increments across the BSJ of $\operatorname{circPOLR} 2 A$ or $\operatorname{circPVT1}$ (Fig. 1h; Extended Data Fig. 3a-b). Only BSJ-gRNAs could guide RfxCas13d to knock down these circRNAs without affecting linear RNA, whereas other non-BSJ site containing gRNAs could direct RfxCas13d to degrade both circular and linear RNAs but to a lesser degree (Fig. 1h; Extended Data Fig. 3a-b).

We examined the position-effect of the BSJ-gRNA spacer for RfxCas13d-mediated circRNA knockdown at the single nucleotide resolution by tiling gRNAs across the BSJ of $\operatorname{circPOLR} 2 A$. We observed that gRNA spacers with the BSJ in the center ( $-7 \mathrm{nt} \sim 7 \mathrm{nt}$ spanning the BSJ site) exhibited high knockdown efficiency without affecting linear cognate RNAs (Fig. 1h; Extended Data Fig. 3c). Similar results were observed for two other circRNAs circHIPK3 (1,099 nt) and circRTN4 by tiling assays (Extended Data Fig. 3d-e). The advantage of the RfxCas13d system in mediating circular but not linear RNA interference (Fig. 1d, 1h; Extended Data Fig. 3) could be due to distinct biogenesis efficiencies, conformations and turnover rates between circular and linear RNAs ${ }^{7,18}$.

\section{RfxCas13d/BSJ-gRNA screens identify circRNAs that act in a cell-type specific manner in cell proliferation}

Next, we set out to explore the feasibility of using the RfxCas13d/BSJ-gRNA system to perform large-scale, loss-of-function screens of circRNAs in human cells. We constructed a library of BSJ-gRNAs that target highly expressed circRNAs in human cells (Fig. 2a). Among top 10\% highly expressed circRNAs with more than 3 copies in one line of HT29, HeLa, 293FT or H9 cells by using CIRCexplorer2 ${ }^{19}$ (Extended Data Fig. 4a-b), 762 circRNAs were successfully included in the gRNA library (Fig. 2a; Supplementary Table 1). This pool of circRNAs contains the most highly expressed $(>70 \%)$ circRNAs in examined human cell lines (Extended Data Fig. $4 \mathrm{c}$ ) and has a representative length distribution compared to all expressed circRNAs (Extended Data Fig. 4d)

To construct the library, we designed five BSJ-gRNAs that all target sequences 
spanning the BSJ in each circRNA and with different lengths (26 30 nt) (Fig. 2a; Supplementary Table 1). Such BSJ-gRNAs were capable of mediating efficient and specific knockdown for circPOLR2A, circHIPK3, circRTN4 and other examined circRNAs (Fig. 1; Extended Data Fig. 1-3). One paired control gRNA (Fig. 1c) was designed for each circRNA, which is more rigorous than scramble controls commonly used in screening studies (Fig. 2a). The gRNA library was constructed from synthetic oligo pools by Gibson assembly (Fig. 2a). Deep sequencing of enriched gRNAs amplified from the library showed a high representation and uniformity of gRNAs (Extended Data Fig. 4e).

We conducted screens to identify circRNAs that are required for cell proliferation. HT29, HeLa and 293FT cells stably expressing RfxCas13d were infected with the gRNA lentiviral library at a multiplicity of infection (MOI) of 0.3 . These cells were cultured for 30 days followed by gRNA enrichment and deep sequencing (Fig. 2b). Read distributions of two biologically independent experiments showed a high correlation in HT29, HeLa and 293FT cells (Extended Data Fig. 4f-h). After 30 days of culture, some gRNAs were obviously depleted compared to paired control gRNAs in each cell line (Extended Data Fig. 4i-k), indicating these circRNAs might be important for cell growth. We ranked these circRNA candidates according to robust rank aggregation (RRA) scores (Extended Data Fig. 4l-n; Supplementary Table 2), calculated by MAGeCK that evaluates the statistical significance of individual gRNA abundance changes using a negative binomial model, and compared the ranks of gRNAs targeting each circRNA with a null model of uniform distribution ${ }^{20}$ (Extended Data Fig. 5a). A small RRA score indicates a strong negative selection of circRNAs during the screening. In general, we observed mild effects of circRNAs on cell proliferation. By considering low RRA score and high expression level, the top 50 circRNA hits in each cell line were obtained, which were potentially important for cell growth (Extended Data Fig. 41-n; Supplementary Table 3).

We selected $\sim 10$ circRNAs in each cell line for validation by RfxCas13d/BSJ-gRNA. We began with one BSJ-gRNA of each circRNA for validation and the great majority of circRNAs could be successfully knocked down with expected cell growth inhibition in these individual types of cells (Extended Data Fig. 5b; Supplementary Table 4). Among them, circHIPK3 and circKLHL 8 were previously reported to be necessary for cell proliferation by shRNA knockdown ${ }^{21,3}$. To exclude potential off-targets on their parental genes, three BSJ-gRNAs targeting $\operatorname{circHIPK3}$ or $\operatorname{circKLHL} 8$ were designed to deplete these circles and all exhibited high knockdown efficacy and retarded cell proliferation in HeLa or 293FT cells (Extended Data Fig. 6a-c). These phenotypes were unlikely to have resulted from off-target effects on their parental genes which remained unchanged (Extended Data Fig. 6a-c). Their suppression of cell proliferation could be also reproduced by shRNA-mediated knockdown (Extended Data Fig. 6d-f), even though two shRNAs targeting circKLHL8 displayed distinct effects (Extended Data Fig. 6d, 6f), indicating possible off-target effects of these shRNAs.

The majority of circRNA hits ( $>80 \%$ ) were unique to only one examined cell type (Fig. 2c; Extended Data Fig. 7a). This cell-type specific mode of circRNA effects is unlikely due to different expression as they were expressed at similar levels in all three cell lines (Fig. 2d; Extended Data Fig. 7b). Further validation of circRNA hits by RfxCas13d/BSJ-gRNA (Fig. 2e) displayed the consistent observation that knockdown of these circRNAs exhibited a cell-type-specific inhibition of cell growth (Fig. 2f-h). Collectively, these results suggested the feasibility of using RfxCas $13 \mathrm{~d} / \mathrm{BSJ}$-gRNA for 
functional circRNA screening in human cells.

\section{CircFAM120A affects the proliferation of different cell types in vitro and in vivo}

Our screens identified a previously unappreciated circRNA, circFAM120A, which is involved in proliferation of all examined cell types. CircFAM120A is highly expressed with over 20 copies per cell (Extended Data Fig. 8a). Knockdown of $\operatorname{circFAM120A}$ by RfxCas13d with three different BSJ-gRNAs all displayed a retarded cell proliferation in HT29, HeLa and 293FT cells (Fig. 2i-j; Extended Data Fig. 8b). The effect of circFAM120A on cell growth was also seen by shRNA-mediated knockdown (Extended Data Fig. 8c-f).

As RfxCas $13 \mathrm{~d} / \mathrm{BSJ}$-gRNAs could persistently knock down circRNAs, we examined the effect of circFAM120A on cell proliferation in nude mice by introducing HT29 cells stably transfected with the control or RfxCas13d-gRNA plasmids for circFAM120A knockdown. Consistently, two examined gRNAs targeting circFAM120A each showed that circFAM120A is important for cell growth in vivo (Fig. 2k-1). These results also suggest that applicability of RfxCas13d-gRNA for LOF circRNAs in xenograft models.

Next, to identify potential circRNA candidates involved in cell proliferation in vivo, we constructed a new library of BSJ-gRNAs that target 2,908 highly expressed circRNAs in nine human cells (Extended Data Fig. 9a-b; Supplementary Table 5). This pool of circRNAs contains the most highly expressed $(>80 \%)$ circRNAs in examined human tissues (Extended Data Fig. 9c), thus representing a comprehensive pool for screening circRNAs in vivo. We carried out in vivo screens by transferring the BSJ-gRNA libraryinfected RfxCas13d-HT29 cells into nude mice and obtained xenografts 22 days after injection (Extended Data Fig. 9d). RNA-seq analyses revealed that the correlation between two biological repeats in vivo was lower than that in cell screening (Extended Data Fig. 9e). Among the top 200 circRNA hits identified from in vivo screens selected by RRA scores (Fig. 2m; Extended Data Fig. 9f), 15 hits appeared in the top 50 hits in HT29 cells screened by the small gRNA library (Fig. 2a, 2m; Extended Data Fig. 4l). CircFAM120A was among these overlap candidates.

\section{CircFAM120A promotes cell growth by up-regulating its parental gene translation}

To gain insights into the mechanism of $\operatorname{circFAM120A}$ in cell proliferation, we performed transcriptomic analyses after circFAM120A knockdown by BSJ-gRNA or shRNA (Extended Data Fig. 10a). Compared to shRNA-mediated knockdown, the BSJgRNA-mediated knockdown showed a much higher correlation with gene expression (Extended Data Fig. 10b-c), further confirming the specificity and robustness of the RfxCas13d/BSJ-gRNA approach for knocking down circRNAs.

Gene ontology analysis of differentially expressed genes (DEGs) resulting from BSJgRNA-mediated circFAM120A knockdown revealed an enrichment of altered genes involved in cell proliferation and apoptosis (Extended Data Fig. 10d-f). For example, the expression of EIF4EBP1, NUPR1 and $P C K 2$ with reported oncogenic function involved in the AKT pathway ${ }^{22-25}$ was down-regulated upon circFAM120A knockdown (Extended Data Fig. 10g), supporting its role in promoting cell proliferation.

CircFAM120A was mainly localized in the cytoplasm (Extended Data Fig. 11a) with 
few enrichment on polyribosomes (Extended Data Fig. 11b), excluding the possibility of circFAM120A translation. As a few cytoplasmic circRNAs, such as CDRlas and circHIPK3, were found to act as miRNA sponges ${ }^{21,26}$, we asked whether circFAM120A could act in a similar manner. Although predicted miRNA sites of Argonaut 2 (AGO2) binding could be identified computationally from published eCLIP datasets ${ }^{26}$ (Extended Data Fig. 11c-d), RNA Immuno-precipitation (RIP) assays with anti-AGO2 followed by examination of associated RNAs revealed no interaction between circFAM120A and AGO2; whereas other known positive controls including CDR1as and circHIPK3 showed strong association (Extended Data Fig. 11e).

Given that FAM120A is an oncogenic gene related to the AKT pathway ${ }^{27}$ and that AKT pathway was over-represented upon the loss of circFAM120A (Extended Data Fig. 10g).

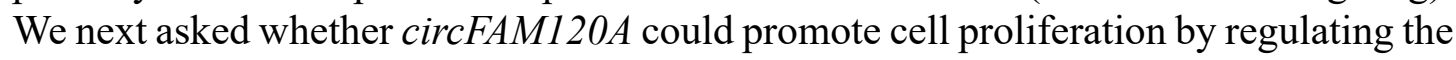
expression of its linear cognate FAM120A mRNA.

First, knockdown of FAM120A mRNA by shRNAs showed an inhibitory effect on 293FT and HT29 cell proliferation (Fig. 3b; Extended Data Fig. 12a-d). Second, knockdown of circFAM120A by RfxCas13d/BSJ-gRNA led to reduced FAM120A protein expression (Fig. 3c; Extended Data Fig. 12e) without affecting FAM120A mRNA level (Fig. 2i), indicating that this reduction might result from inhibited translation of FAM120A mRNA. Consistent with this possibility, polysome profiling showed a shift of linear FAM120A mRNA from polyribosomes to monoribosomes after circFAM120A knockdown by BSJ-gRNA (Fig. 3d-e; Extended Data Fig. 12f), indicating impaired translation. Third, transfection of FAM120A-overexpression vectors in circFAM120A deficient cells fully rescued the impaired cell proliferation (Fig. 3f). These results suggested that circFAM120A promotes cell growth by preventing translation of its cognate linear mRNA.

\section{CircFAM120A prevents FAM120A mRNA from binding to IGF2BP2, leading to efficient translation}

To explore how does $c i r c F A M 120 A$ regulate $F A M 120 A$ mRNA translation, we screened RBPs associated with the circFAM120A-producing locus from published eCLIP databases (https://www.encodeproject.org/), and identified IGF2BP2, an RNA binding protein with reported function in cell proliferation by suppressing mRNA translation 28,29 (Fig. 3a). Polysome profiling revealed distinct distributions of IGF2BP2, circFAM120A and the cognate linear FAM120A mRNA (Fig. 3g; Extended Data Fig. $12 \mathrm{~g}$ ). In agreement with its inhibitory function in translation, $>95 \%$ IGF2BP2 was localized to the ribosome-free fraction, where both circular and linear FAM120A RNAs were absent. The majority of circFAM120A presented in the light monoribosome fraction, where $<5 \%$ IGF2BP 2 was enriched (Extended Data Fig. 12g); in contrast, only $\sim 10 \%$ of FAM120A mRNAs were localized to monoribosomes and most FAM120A mRNAs were associated with polyribosomes in the heavy ribosome fraction (Fig. 3g; Extended Data Fig. 12g). These findings indicated that circFAM120A might compete with its cognate mRNA for IGF2BP2 binding in monoribosomes and that the IGF2BP2unbound-FAM120A mRNA could be engaged with polyribosomes for translation. To confirm this hypothesis, we performed a series of RNA pull-down and IGF2BP2 RIP assays. The strong and specific association of IGF2BP2 with circFAM120A, but not other circRNAs, was observed (Fig. 3h-i). Knockdown of circFAM120A led to enhanced interaction between linear FAM120A mRNA and IGF2BP2 in HT29 cells 
(Fig. 3j) and 293FT cells (Extended Data Fig. 12h). In vitro competition assays with IGF2BP2 immunoprecipitated from 293FT cells or purified from E. coli both showed that the addition of circFAM120A strikingly impaired IGF2BP2 and FAM120A mRNA interaction (Fig. 3k-1). Thus, although sharing the same sequences with circFAM120A, the linear mRNA showed much weaker interaction with IGF2BP2 than circFAM20A (Fig. 3h-i). These results are consistent with a role of circFAM120A in promoting human cell proliferation by preventing FAM120A mRNA from binding to IGF2BP2 for efficient translation (Fig. 3m).

\section{Application of the RfxCas13d/BSJ-gRNA system to identify key circRNAs important for the development of mouse embryos}

It has been shown that circRNAs are abundant in early mouse embryos from the zygote to blastocyst stages ${ }^{30}$. However, it remains unknown whether any circRNA is important during development. To identify such circRNAs, we first explored whether the RfxCas13d/BSJ-gRNA system is applicable to mouse embryos. To do this, we microinjected purified RfxCas13d mRNAs and guide RNAs targeting Kras and Brg1 mRNAs into zygotes, followed by the examination of knockdown efficiency of each mRNA and defects in the preimplantation stage embryos (Fig. 4a). RfxCas13d/gRNAs displayed efficient knockdown of both Kras and Brgl mRNAs (Extended Data Fig. 13a). Only knockdown of $\mathrm{Brgl}$, but not Kras, led to reduced blastocyst formation as previously reported ${ }^{31,32}$ (Extended Data Fig. 13b-d), suggesting the specificity and nontoxicity of using this system to study RNA function in mouse embryos.

Next, we applied the RfxCas13d/BSJ-gRNA system to screen potentially functional circRNAs during preimplantation development of mouse embryos. We selected a pool of 24 mouse circRNAs containing 15 circRNAs obtained from our screens in human cell lines (Fig. 2c) with mouse homolog circRNA expression in examined mouse cells and another nine highly expressed and conserved circRNAs from mouse preimplantation embryos ${ }^{30}$ (Fig. 4b). We confirmed their high expression during mouse preimplantation development (Supplementary Table 6). Initial screening by injecting RfxCas $13 \mathrm{~d}$ mRNA with one gRNA each targeting 24 individual circRNAs into more than 30 50 mouse zygotes identified one circRNA (circManla2) as a potential candidate important for mouse embryo preimplantation development (Supplementary Table 6).

CircMan la 2 is a conserved circRNA that is derived from exons 2-6 of its parental gene in mouse or in human (Extended Data Fig. 14a). To exclude any potential off-target, one paired control and another BSJ-gRNA for circMan la 2 were also tested. Consistent with results obtained in human cell lines (Fig. 1 and Extended Data Fig. 1-2), RfxCas13d efficiently targeted circMan1a2 with BSJ-gRNAs with little effect on its cognate mRNA in mouse embryos (Fig. 4d). Importantly, microinjection of RfxCas13d mRNA and BSJ-gRNAs targeting circManla2 into mouse zygotes led to retarded embryonic development as shown by decreased blastocyst formation rate (Fig. 4e) and smaller blastocoel according to the diameter and area of cavity (Fig. 4f-g; Extended Data Fig. 14b). As controls, knockdown other circRNAs, such as circDcbld2, had no obvious phenotype (Extended Data Fig. 14c; Supplementary Table 7). Together, these screens indicated potentially regulatory roles of circRNAs in the preimplantation stage of mouse embryos. 


\section{Discussion}

Due to the complete sequence overlap between back-spliced RNA circles and their linearly spliced RNA isoforms except BSJs, the functional study of individual circRNAs has been impeded by the lack of tools uniquely targeting these circles. We have shown that the RfxCas13d/BSJ-gRNA system achieves precise and robust circRNA-specific knockdown without disturbing their linear cognate mRNAs (Fig. 1ad) and that this system is applicable at both individual and genome-wide levels (Fig. 2a-b). The Cas13 approach is unique to study LOF of circRNAs, which are derived from the middle exons of genes ${ }^{33}$. There are at least two reasons that account for this. First, the overall conformation of circRNAs is more stable and rigid than that of linear RNAs $^{18}$. Thus, once targeted by gRNA and Cas 13 in a circRNA, such rigid structures would be continuously to be targeted by this system; whereas in a linear RNA, the highly dynamic and flexible folding status theoretically make it difficult for effective targeting. Second, back-splicing efficiency is extremely slow, which is less than $1 \%$ of that of the canonical splicing in cells ${ }^{7}$. Once cleaved by Cas13, the newly produced circRNAs cannot compensate for the loss of circRNAs, leading to a consistent reduction of circRNAs by this system.

Screening of circRNAs involved in cell growth in different human cells suggests the cell-type specific manner of action of circRNAs, identifying only a few that likely act in examined human cells (Fig. 2a-h). This observation is consistent with a recent finding that knockdown of long noncoding RNAs can affect gene expression in a cell typespecific manner as revealed by CRISPRi-based genome-scale screening ${ }^{34}$. In the case of circRNAs, considering their species- ${ }^{35}$, tissue- and cell type-specific expression patterns ${ }^{36,7,37}$, our results indicate that the great majority of circRNAs may not play generic housekeeping roles but some may act in a regulatory fashion in a cell typespecific manner.

Although most act in the cell-specific manner, it is worthwhile noting that our screens identified one commonly-expressed oncogenic circRNA, circFAM120A, which is derived from the FAM120A gene with reported roles in cancer ${ }^{27}$. CircFAM120A promotes cell proliferation both in vitro and in vivo (Fig. 2i-1). CircFAM120A is largely localized to monoribosomes (Fig. $3 \mathrm{~d}, 3 \mathrm{~g}$ ), where it interacts with translation inhibitor IGF2BP2 ${ }^{28,29}$ to prevent the binding between IGF2BP2 and FAM120A mRNA, facilitating the IGF2BP2-unboud mRNA translation (Fig. 3g-m).

The RfxCas13d/BSJ-gRNA system could also be used to effectively and robustly disturb linear and circular RNA expression in mouse embryos (Fig. 4a-d; Supplementary Tables 6-7). Screening a small pool of conserved circRNAs in mouse embryos identified circManla2 that is important for mouse embryo preimplantation, suggesting previously unknown functional relevance of circRNAs in the early animal development. Generation of circRNA loss-of-function mouse models without affecting their parental mRNA expression will be a necessity to prove the physiological importance of endogenous circRNAs. Nevertheless, future application of the RfxCas $13 \mathrm{~d} / \mathrm{BSJ}$-gRNA system will likely enable the discovery of circRNAs that participate in other biological processes.

\section{References:}


1. Li, X., Yang, L. \& Chen, L. L. The Biogenesis, Functions, and Challenges of Circular RNAs. Mol. Cell 71, 428-442 (2018).

2. Kristensen, L. S. et al. The biogenesis, biology and characterization of circular RNAs. Nat. Rev. Genet. 20, 675-691 (2019).

3. Chen, S. et al. Widespread and Functional RNA Circularization in Localized Prostate Cancer. Cell 176, 831-843 e822 (2019).

4. Guarnerio, J. et al. Oncogenic Role of Fusion-circRNAs Derived from CancerAssociated Chromosomal Translocations. Cell 166, 1055-1056 (2016).

5. Legnini, I. et al. Circ-ZNF609 Is a Circular RNA that Can Be Translated and Functions in Myogenesis. Mol. Cell 66, 22-+ (2017).

6. Piwecka, M. et al. Loss of a mammalian circular RNA locus causes miRNA deregulation and affects brain function. Science 357 (2017).

7. Zhang, Y. et al. The Biogenesis of Nascent Circular RNAs. Cell Rep. 15, 611624 (2016).

8. Xia, P. et al. A Circular RNA Protects Dormant Hematopoietic Stem Cells from DNA Sensor cGAS-Mediated Exhaustion. Immunity 48, 688-701 e687 (2018).

9. Abudayyeh, O. O. et al. C2c2 is a single-component programmable RNAguided RNA-targeting CRISPR effector. Science 353 (2016).

10. East-Seletsky, A. et al. Two distinct RNase activities of CRISPR-C2c2 enable guide-RNA processing and RNA detection. Nature 538, 270-273 (2016).

11. Konermann, S. et al. Transcriptome Engineering with RNA-Targeting Type VID CRISPR Effectors. Cell 173 (2018).

12. Shmakov, S. et al. Discovery and Functional Characterization of Diverse Class 2 CRISPR-Cas Systems. Mol. Cell 60, 385-397 (2015).

13. Abudayyeh, O. O. et al. RNA targeting with CRISPR-Cas13. Nature (2017).

14. Li, L., Zhao, H., Chen, Z., Mu, X. \& Guo, L. Aptamer biosensor for label-free square-wave voltammetry detection of angiogenin. Biosens. Bioelectron. 30, 261-266 (2011).

15. Cox, D. B. T. et al. RNA editing with CRISPR-Cas13. Science, 358, 1019-1027 (2017).

16. Hansen, T. B. et al. miRNA-dependent gene silencing involving Ago2-mediated cleavage of a circular antisense RNA. EMBO J. 30, 4414-4422, doi:10.1038/emboj.2011.359 (2011).

17. Chen, G. W., Shi, Y. T., Zhang, Y. \& Sun, J. Y. CircRNA_100782 regulates pancreatic carcinoma proliferation through the IL6-STAT3 pathway. Onco. Targets Ther. 10, 5783-5794 (2017).

18. Liu, C. X. et al. Structure and Degradation of Circular RNAs Regulate PKR Activation in Innate Immunity. Cell 177, 865-880 e821 (2019).

19. Zhang, X. O. et al. Diverse alternative back-splicing and alternative splicing landscape of circular RNAs. Genome Res. 26, 1277-1287 (2016).

20. Li, W. et al. MAGeCK enables robust identification of essential genes from genome-scale CRISPR/Cas9 knockout screens. Genome Biol. 15, 554 (2014).

21. Zheng, Q. P. et al. Circular RNA profiling reveals an abundant circHIPK3 that regulates cell growth by sponging multiple miRNAs. Nat. Commun. 7, 11215 (2016).

22. Lee, M., Kim, E. J. \& Jeon, M. J. MicroRNAs 125a and 125b inhibit ovarian cancer cells through post-transcriptional inactivation of EIF4EBP1. Oncotarget 7, 8726-8742 (2016).

23. Mu, Y. C. et al. NUPR1 maintains autolysosomal efflux by activating SNAP25 transcription in cancer cells. Autophagy 14, 654-670 (2018). 
24. Vincent, E. E. et al. Mitochondrial Phosphoenolpyruvate Carboxykinase Regulates Metabolic Adaptation and Enables Glucose-Independent Tumor Growth. Mol. Cell 60, 195-207 (2015).

25. Wallin, J. J. et al. Nuclear phospho-Akt increase predicts synergy of PI3K inhibition and doxorubicin in breast and ovarian cancer. Sci. Transl. Med. 2, 48ra66 (2010).

26. Memczak, S. et al. Circular RNAs are a large class of animal RNAs with regulatory potency. Nature 495, 333-338 (2013).

27. Bartolome, R. A. et al. IL13 Receptor alpha 2 Signaling Requires a Scaffold Protein, FAM120A, to Activate the FAK and PI3K Pathways in Colon Cancer Metastasis. Cancer Res. 75, 2434-2444 (2015).

28. Dai, N. et al. IGF2BP2/IMP2-Deficient Mice Resist Obesity through Enhanced Translation of Ucp1 mRNA and Other mRNAs Encoding Mitochondrial Proteins. Cell Metab. 21, 609-621 (2015).

29. Li, Z. et al. An HMGA2-IGF2BP2 axis regulates myoblast proliferation and myogenesis. Dev. Cell 23, 1176-1188 (2012).

30. Fan, X. et al. Single-cell RNA-seq transcriptome analysis of linear and circular RNAs in mouse preimplantation embryos. Genome Biol. 16, 148 (2015).

31. Bultman, S. et al. A Brg1 null mutation in the mouse reveals functional differences among mammalian SWI/SNF complexes. Mol. Cell 6, 1287-1295 (2000).

32. Bultman, S. J. et al. Maternal BRG1 regulates zygotic genome activation in the mouse. Genes Dev. 20, 1744-1754 (2006).

33. Zhang, X. O. et al. Complementary sequence-mediated exon circularization. Cell 159, 134-147 (2014).

34. Liu, S. J. et al. CRISPRi-based genome-scale identification of functional long noncoding RNA loci in human cells. Science 355 (2017).

35. Dong, R., Ma, X. K., Chen, L. L. \& Yang, L. Increased complexity of circRNA expression during species evolution. RNA Biol. 14, 1064-1074 (2017).

36. Salzman, J., Chen, R. E., Olsen, M. N., Wang, P. L. \& Brown, P. O. Cell-type specific features of circular RNA expression. PLoS Genet. 9, e1003777 (2013).

37. Rybak-Wolf, A. et al. Circular RNAs in the Mammalian Brain Are Highly Abundant, Conserved, and Dynamically Expressed. Mol. Cell 58, 870-885 (2015).

\section{Acknowledgments:}

We thank Chen and Yang laboratories for discussion. This work was supported by the Chinese Academy of Sciences (CAS) (XDB19020104), the National Natural Science Foundation of China (NSFC) (31725009, 31821004, 31730111, 31730062), the Shanghai Municipal Commission for Science and Technology (19411951800) and the Howard Hughes Medical Institute International Program (55008728).

\section{Author contributions:}

L.-L.C. supervised and conceived the project. L.-L.C., L.Y., J.L., S.-Q.L., X.L., L.Z., and W.X. designed experiments. L.Z. performed circRNA screening in mouse embryos supervised by J.L; S.-Q.L., X.L., L.Z., S.-M.C., L.-Z.Y., J.-L.Z., X.G., S.-K.G. and J.W. performed all other experiments; W.X., Y.-N.L., L.Y. preformed computational 
analyses. L.-L.C. and L.Y. wrote the paper with input from S.-Q.L., X.L., W.X and J.L.

\section{Competing interests:}

The authors declare that they have no competing financial interests.

\section{Supplementary Materials:}

Materials and methods

Extended Data Fig 1-14

Supplementary Tables S1-S9 


\section{Figures and Figure Legends}

a

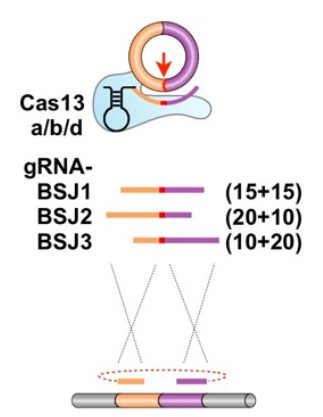

b

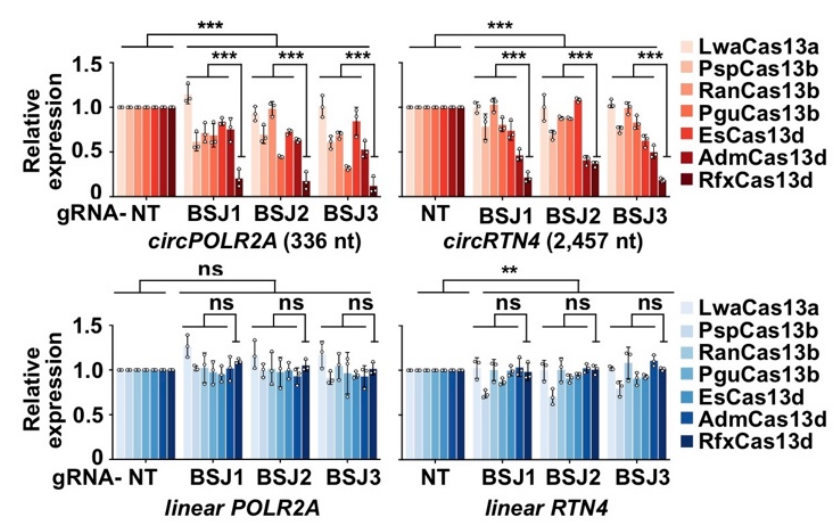

C

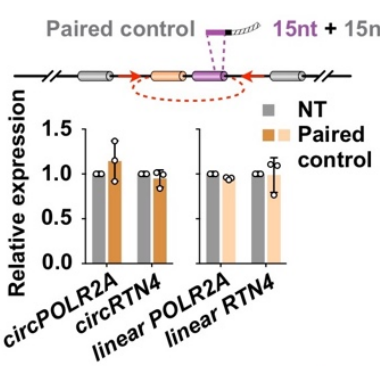

e

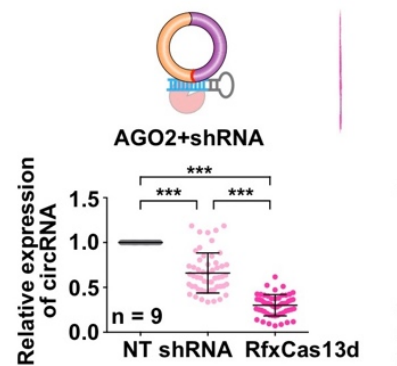

g
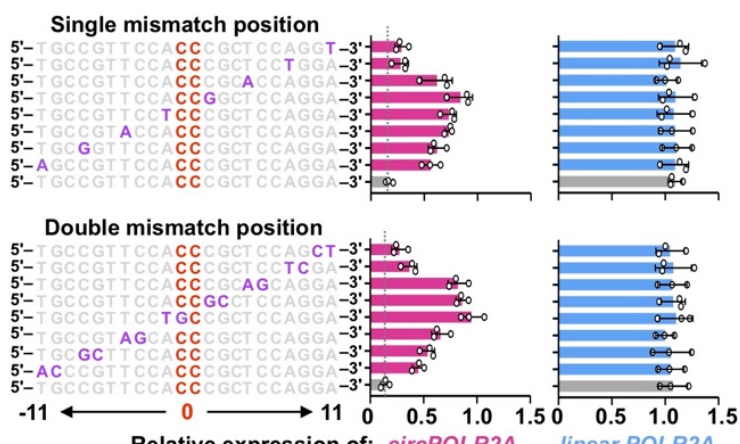

Relative expression of: circPOLR2A linear POLR2

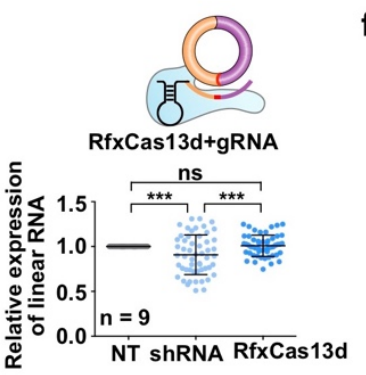

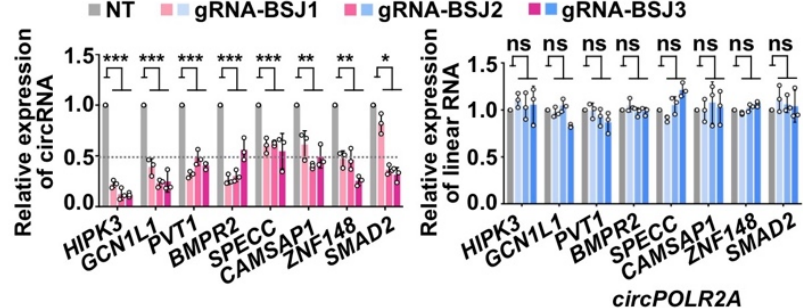

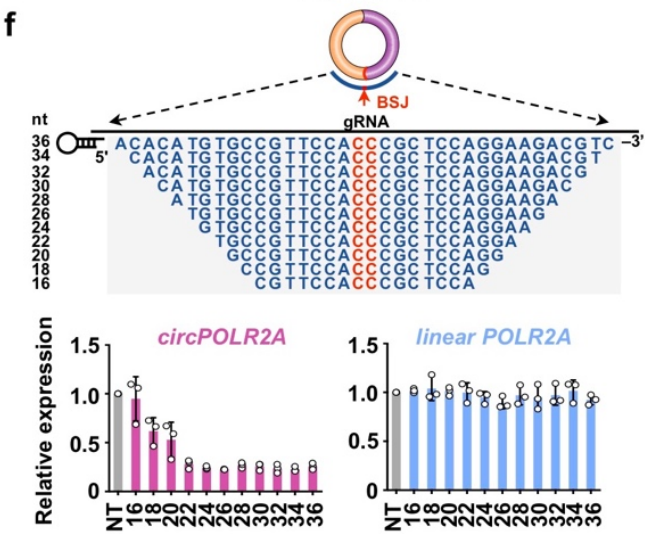

h

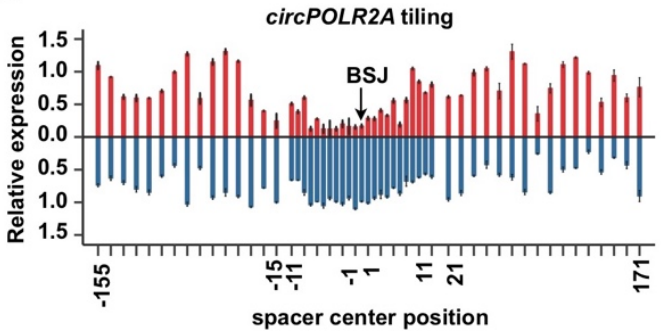

Fig. 1 | RfxCas13d/BSJ-gRNA discriminates circRNAs from mRNAs.

a. Schematic of circRNA knockdown by Cas13 orthologues. Three guide RNAs targeting the back-splicing junction site (red arrow) were designed for each circRNA (BSJ-gRNAs).

b. Evaluation of different Cas13-protein-mediated knockdown on circRNAs. Expression levels of two circRNAs, $\operatorname{circPOLR} 2 \mathrm{~A}$ and $\operatorname{circRTN4}$, and their cognate 
mRNAs were detected by qRT-PCR in 293FT cells. NT, non-targeting guide RNA.

c. A paired control gRNA with partial sequences replaced by scrambled sequences for circPOLR $2 A$ or circRTN4 did not guide RfxCas $13 \mathrm{~d}$ to affect expression of circRNAs or mRNAs.

d. The RfxCas13d/BSJ-gRNA system mediates specific and robust knockdown of randomly selected circRNAs, but not their cognate linear mRNAs in 293FT cells.

e. The RfxCas 13d/BSJ-gRNA system exhibits higher specificity and efficiency than shRNAs on circRNA knockdown. Knockdown of nine circRNAs with RfxCas13d/BSJgRNAs or position-matched shRNAs was compared. Each circRNA was targeted by two BSJ-gRNAs and two position-matched shRNAs; the expression of each cognate linear mRNA was also examined.

f. Minimal length requirement of BSJ-gRNA spacer for efficient RfxCas13d knockdown. Top, lengths and sequences of gRNA spacers flanking the $\operatorname{circPOLR} 2 \mathrm{~A}$ BSJ site; bases flanking the BSJ site are shown in red. Bottom, knockdown efficiencies of $\operatorname{circPOLR} 2 A$ and linear POLR $2 A$ by each BSJ-gRNA and RfxCas $13 \mathrm{~d}$ were detected by qRT-PCR.

g. Mismatch tolerance of RfxCas $13 \mathrm{~d}$ for circRNA targeting. Left, guides containing single or double mismatches at varying positions across spacer sequences for circPOLR $2 A$ are shown; mismatch positions are shown in purple; bases flanking the BSJ site are shown in red. Right, knockdown efficiencies of $\operatorname{circPOLR} 2 A$ and linear $P O L R 2 A$ by each BSJ-gRNA and RfxCas $13 \mathrm{~d}$ were detected by qRT-PCR.

h. Efficiency and specificity of RfxCas $13 \mathrm{~d}$ for circRNA and cognate linear mRNA

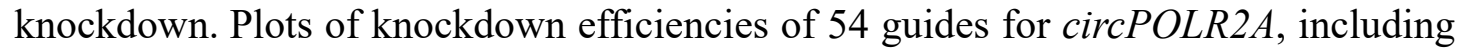
23 guides tiled across the BSJ and 31 guides tiled on the overlap region of $\operatorname{circPOLR} 2 \mathrm{~A}$ and linear POLR2A.

All transcript levels were normalized to $A C T B$, and values represent mean $+/-\mathrm{SD}$ with $n=3, *: P<0.05$; **: $P<0.01$; **: $P<0.001$; ns, not significant. 


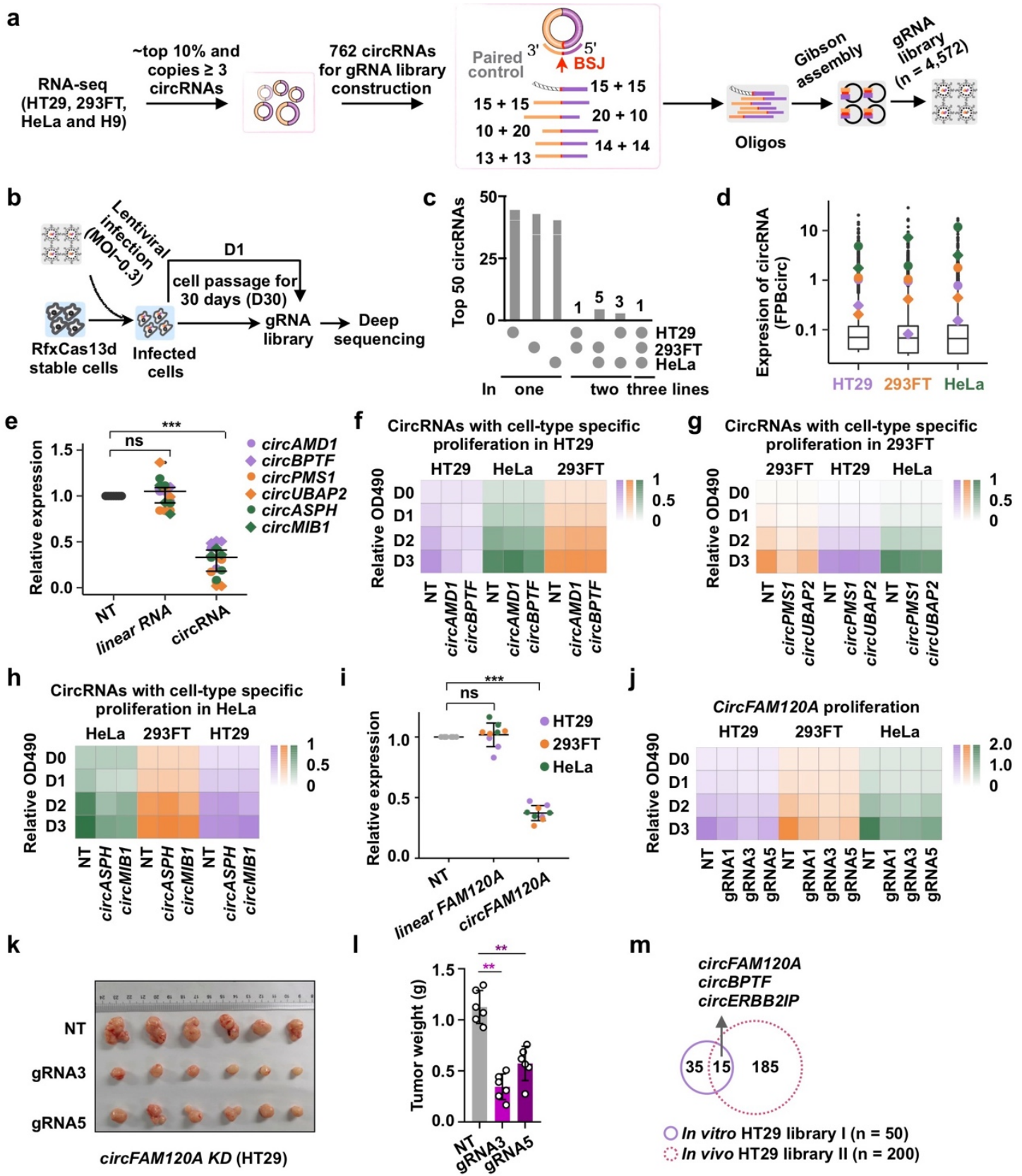

Fig. 2 | BSJ-gRNA library construction and circRNAs act in a cell-type specific manner in cell proliferation.

a. Schematic of gRNA library design and construction. One paired control gRNA $(\mathrm{n}=762)$ and five BSJ-gRNAs (circRNA gRNAs, $\mathrm{n}=3,810$ ) were designed for each candidate circRNA. In total, 4,572 gRNAs were included in the library.

b. Screen of circRNAs necessary for cell growth and proliferation. The gRNA lentiviral library was individually delivered into HT29, 293FT and HeLa cells stably expressing RfxCas 13d. Infected cells were collected at day 1 (D1) and day 30 (D30) after infection and genomic DNA was extracted for gRNA amplification and deep sequencing.

c. Overlapping analysis of the top50 circRNA hits that potentially have impacts on cell growth by screens in HT29, 293FT and HeLa cells.

d. Expression (shown by FPBcirc) of circRNAs that exhibit cell type-specific effect on cell proliferation in HT29, 293FT and HeLa cells.

e. Knockdown of circRNAs shown in (d) by the RfxCas13d/BSJ-gRNA system in HT29, 293FT and HeLa cells, individually. Expression levels of circRNAs and linear 
mRNAs were detected by qRT-PCR. All transcript levels were relative to $A C T B$, and values represent mean $+/-\mathrm{SD}$ with $n=3$, ***: $P<0.001$; ns, not significant.

f. Cell type- specific growth effect of $\operatorname{circ} A M D 1$ and $\operatorname{circBPTF}$ in HT 29 cells, shown by MTT cell proliferation assays.

g. Cell type- specific growth effect of circPMS1 and circUBAP2 in 293FT cells, shown by MTT cell proliferation assays.

h. Cell type- specific growth effect of $\operatorname{circASPH}$ and $\operatorname{circMIB1}$ in HeLa cells, shown by MTT cell proliferation assays.

i. Specific knockdown of circFAM120A by RfxCas13d/ BSJ-gRNAs in HT29, 293FT and HeLa cells. Expression levels of circFAM120A and linear FAM120A were detected by qRT-PCR. All transcript levels were relative to $A C T B$, and values represent mean $+/$ - SD with $n=3$, ***: $P<0.001$; ns, not significant.

j. Knockdown of circFAM120A by RfxCas13d/ BSJ-gRNAs inhibits HT29, 293FT and HeLa cell growth and proliferation, as revealed by MTT cell proliferation assays.

k-l. Knockdown of circFAM120A by RfxCas13d inhibits tumors growth. The image of tumors from nude mice injected subcutaneously with NT and circFAM120A-KD HT29 cells is shown in (k), and the statistics of tumor weight is shown in (l).

m. Overlap of circRNA candidates between in cells (top 50, $\mathrm{n}=762$ ) and in vivo (top 200, $\mathrm{n}=2,908)$ screens in HT29 cells. CircFAM120A was identified in both screens. 
a

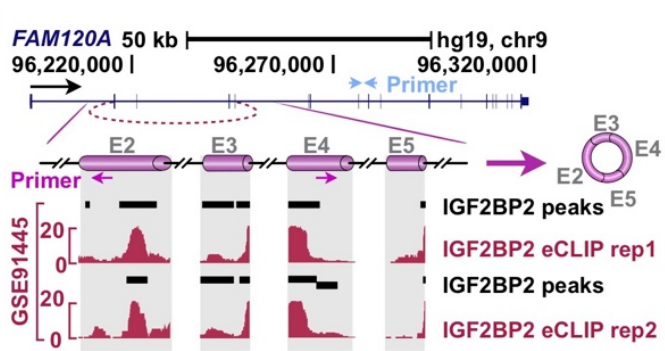

b

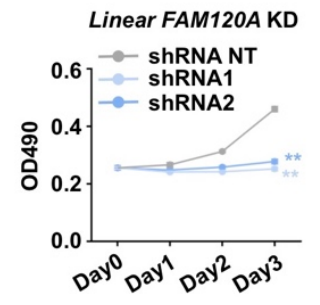

C

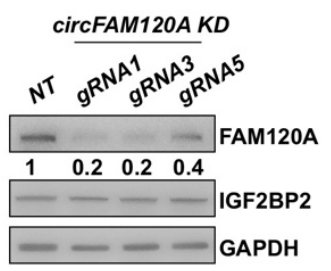

d

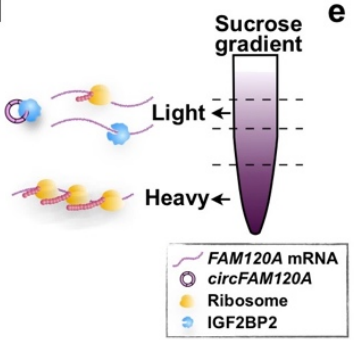

h

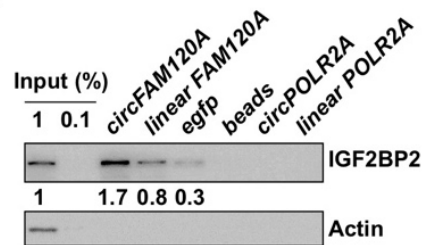

k

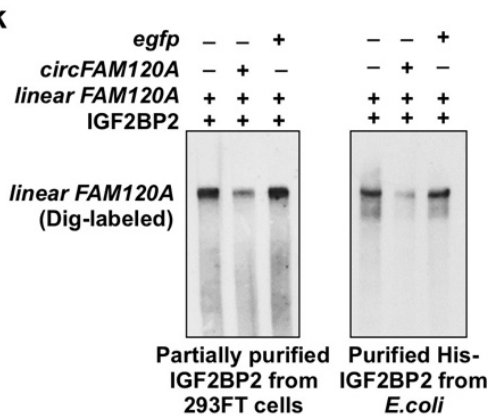

f

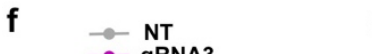

g

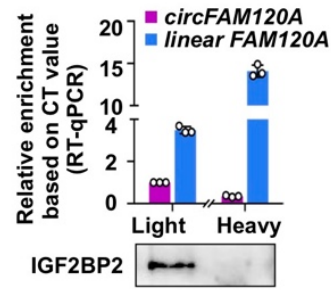

j
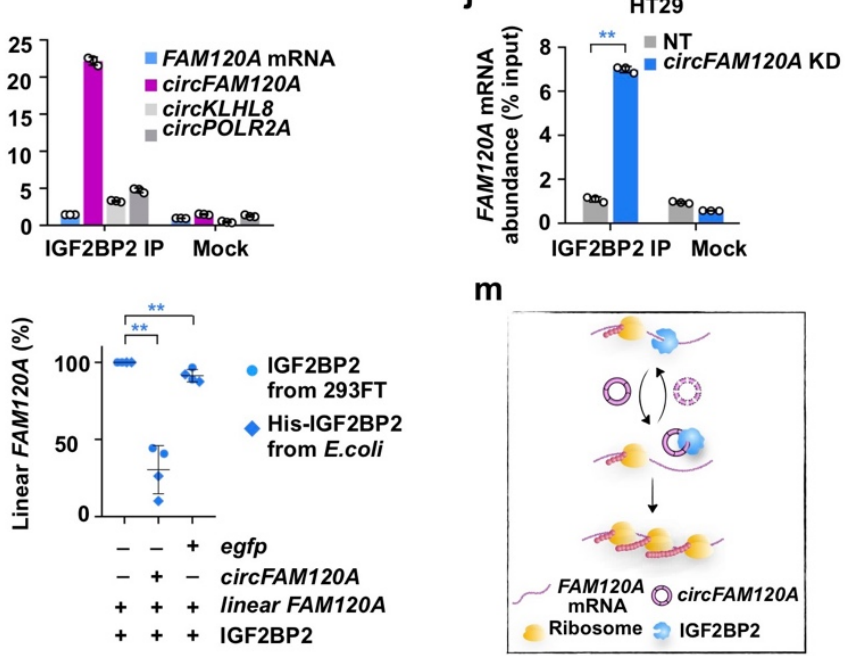

m

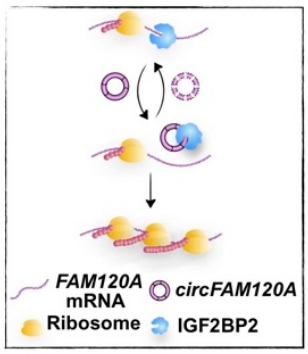

Fig. 3 | CircFAM120A promotes cell proliferation by preventing $F A M 120 A$ mRNA from interacting with IGF2BP2 for efficient translation.

a. Prediction of IGF2BP2-binding peaks in circFAM120A. Top, genomics locus and diagram of linear FAM120A and circFAM120A (shown as cylinders in magenta). Blue and magenta arrows indicate location of primer for linear FAM120A or circFAM120A, respectively. Bottom, IGF2BP2-binding peaks and wiggle-tracks of eCLIP-seq data in K562 cells (GEO, GSE91445) revealed that IGF2BP2 proteins were targeted on circFAM120A producing circularized exons.

b. Knockdown of FAM120A mRNA by shRNAs inhibits 293FT cells growth and proliferation, as revealed by MTT cell proliferation assays.

c. Knockdown of circFAM120A by RfxCas13d/ BSJ-gRNAs

leads to reduced expression of FAM120A protein in 293FT cells.

d. Schematic of the sucrose gradient used to segregate light and heavy fraction in polysome profiling assay.

e. Knockdown of circFAM120A leads to reduced enrichment of linear FAM120A on polyribosomes (heavy fraction). Cytoplasmic extracts from NT or circFAM120A-KD 293FT cells, were loaded on 10\%-45\% sucrose gradients. Enrichments of FAM120A mRNA in light or heavy fraction was measured by qRT-PCR. All values represent mean $+/-\mathrm{SD}$ with $n=3, * *: P<0.01$. 
f. Overexpression of linear FAM120A mRNA rescues the impaired cell proliferation in circFAM120A deficient cells, as revealed by MTT cell proliferation assays. All values represent mean $+/-\mathrm{SD}$ with $n=3, * *: P<0.01$.

g. The distribution of circFAM120A, linear FAM120A and IGF2BP2 protein in the light or heavy fraction of $293 \mathrm{FT}$ cells, as revealed by polysome profiling assays.

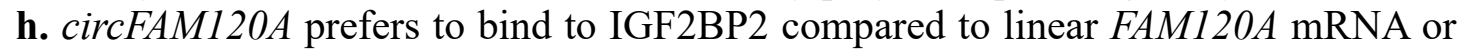
other negative controls, as revealed by biotin-labeled RNA pull-down assays.

i. IGF2BP2 perfers to bind circFAM120A, compared to linear FAM120A mRNA or other circRNAs, as revealed by IGF2BP2 RNA immunoprecipitation (RIP) in 293FT cells. The abundance of RIP-enriched RNAs was measured by qRT-PCR. All values represent mean $+/-\mathrm{SD}$ with $n=3, * *: P<0.01$.

j. Knockdown of circFAM120A leads to increased interaction between cognate FAM120A mRNA and IGF2BP2 in HT29 cells. The abundance of FAM120A mRNA was measured by qRT-PCR, and values represent mean $+/$ SD with $n=3, *: P<0.05$; $* *: P<0.01$.

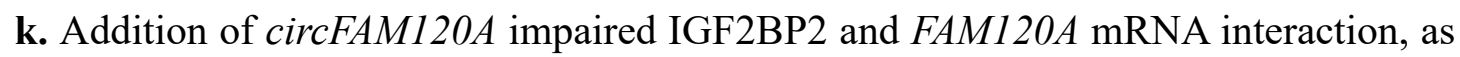
revealed by in vitro competition assay. The IGF2BP2 protein is immunoprecipitated from 293FT cells (left) or purified from the E. coli (right).

1. Quantification of in vitro competition assays shown in (K). Data are shown as median and IQR. n.s., $\mathrm{p}>0.05,{ }^{*} \mathrm{p}<0.01$, Student's t test.

m. A proposed model of $\operatorname{circFAM120A}$ binding IGF2BP2 to prevent linear FAM120A mRNA from interaction with IGF2BP2 for an efficient translation to promote cell growth. See text for details. 
a

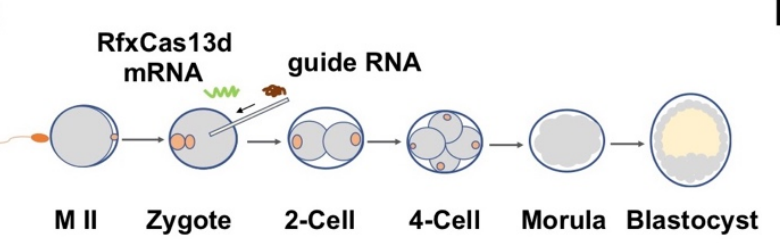

b

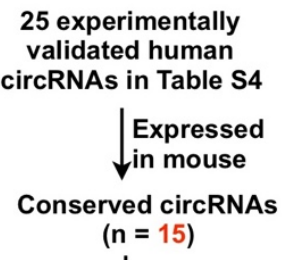

c

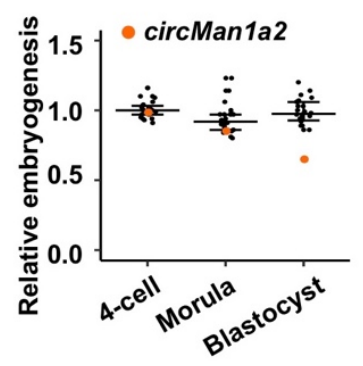

$\mathbf{e}$

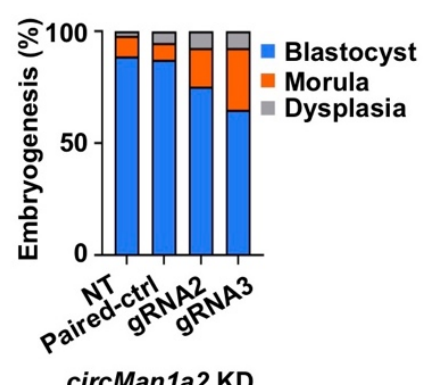

d

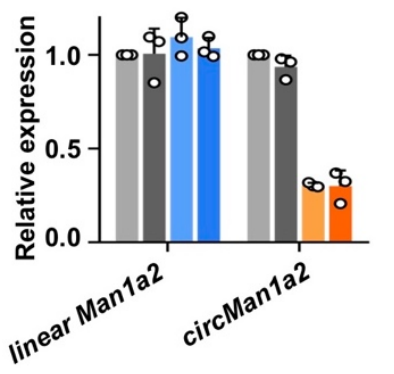

f

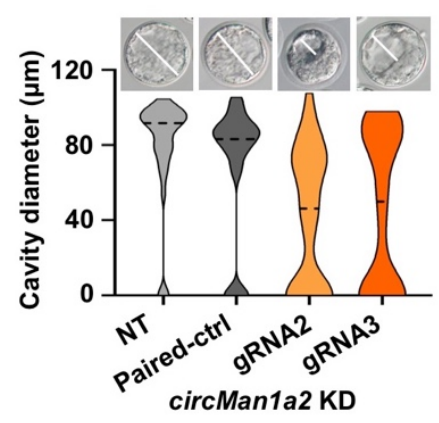

Top 10 circRNAs in mouse preimplantation embryo

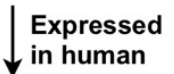

Conserved circRNAs $(n=9)$
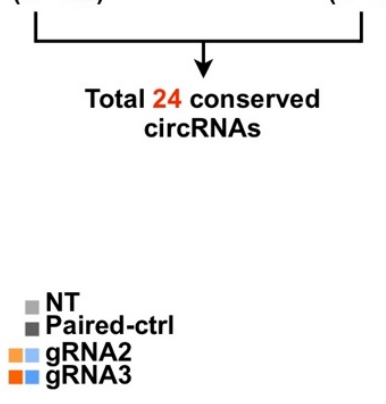

g

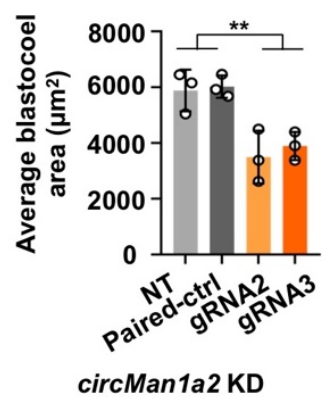

Fig. 4 | Screening circRNAs with functional potential during mouse preimplantation development with RfXCas13d/BSJ-gRNA.

a. Schematic of RfxCas13d mRNA (green curve) and gRNA (brown curve) microinjection into mouse zygotes for RNA knockdown during preimplantation development.

b. A schematic workflow to show the strategy of selecting 24 circRNAs tested in the preimplantation development of mouse embryos. The expression data of circRNAs in mouse preimplantation embryo from GEO: GSE53386 $6^{30}$.

c. The effect of embryogenesis of 24 circRNA candidates knockdown individually by RfxCas13d/BSJ-gRNA during mouse preimplantation development.

d. Knockdown of circManla2 by RfxCas13d/BSJ-gRNAs in zygotes. Expression levels of circMan $1 a 2$ and linear Manla 2 were detected by qRT-PCR. All transcript levels were relative to Gapdh, and values represent mean $+/-$ SD with $n=3$.

e. Knockdown of circMan $1 \mathrm{a} 2$ in zygotes led to reduced blastocyst formation $72 \mathrm{~h}$ after microinjection of RfxCas13d mRNA and the corresponding BSJ-gRNAs into mouse zygotes.

f-g. Cavity diameter (f) and area (g) of blastocysts were measured at $72 \mathrm{~h}$ after microinjection of RfxCas13d mRNA and circManla2 BSJ-gRNAs into mouse zygotes. Bright-field images showed the representative cavity diameter of blastocyst in each group (f). The blastocoel area was calculated as length $\times$ width. All values represent mean $+/$ - SD with $n=3, * *: P<0.01$. 


\section{Supplemental Information}

\section{Screening for functional circular RNAs in human cells using the CRISPR-Cas13 system}

Siqi Li ${ }^{1,4}$, Xiang Li ${ }^{1,4}$, Wei Xue ${ }^{2,4}$, Lin Zhang ${ }^{1,4}$, Shi-Meng Cao ${ }^{1}$, Yun-Ni Lei ${ }^{2,3}$, Liang-Zhong Yang ${ }^{1}$, Si-Kun Guo ${ }^{1}$, Jia-Lin Zhang ${ }^{2}$, Xiang Gao ${ }^{1,3}$, Jia Wei ${ }^{2}$, Jinsong $\mathrm{Li}^{1,3 *}$, Li Yang ${ }^{2,3, *}$, Ling-Ling Chen ${ }^{1,3, *}$

${ }^{1}$ State Key Laboratory of Molecular Biology, Shanghai Key Laboratory of Molecular Andrology, CAS Center for Excellence in Molecular Cell Science, Shanghai Institute of Biochemistry and Cell Biology, University of Chinese Academy of Sciences, Chinese Academy of Sciences, 320 Yueyang Road, Shanghai 200031, China

${ }^{2}$ CAS Key Laboratory of Computational Biology, CAS-MPG Partner Institute for Computational Biology, Shanghai Institute of Nutrition and Health, Shanghai Institutes for Biological Sciences, University of Chinese Academy of Sciences, Chinese Academy of Sciences, 320 Yueyang Road, Shanghai 200031, China

${ }^{3}$ School of Life Science and Technology, ShanghaiTech University, 100 Haike Road, Shanghai 201210, China

${ }^{4}$ These authors contributed equally

* Correspondence should be addressed to L.Y. (liyang@picb.ac.cn), J. L. (jsli@sibcb.ac.cn) and L.-L.C. (linglingchen@sibcb.ac.cn). 


\section{Materials and Methods}

\section{Cell culture}

HT29, HeLa and 293FT cells were purchased from the American Type Culture Collection (ATCC; http://www.atcc.org) or ThermoFisher, respectively, and were originally authenticated using STR profiling. HT29 cells were maintained in RPMI 1640, HeLa (human female origin) and 293FT cells (human fetus origin) were maintained in DMEM, and all supplemented with 10\% Fetal Bovine Serum (FBS) and $0.1 \%$ penicillin/streptomycin. We maintained cell lines at $37^{\circ} \mathrm{C}$ in a $5 \% \mathrm{CO}_{2}$ cell culture incubator and tested all cell lines routinely to exclude mycoplasma contamination.

\section{Plasmid construction and transfection}

For construction of Cas 13 expression vectors, human codon-optimized DNA sequences of seven Cas13 orthologues (LwaCas13a, PspCas13b, PguCas13b, RanCas13b, EsCas13d, AdmCas13d and RfxCas13d) were synthesized in Genscript company and individually cloned into the $\mathrm{p} 23$-phage vector containing sequences for a C-terminal fused msfGFP and a Flag tag. DNA sequence of RfxCas $13 \mathrm{~d}$ was further cloned into the p23-phage vector containing sequences for a C-terminal fused mCherry and a Flag tag (p23-RfxCas13d-mcherry-flag). For construction of guide (g)RNA expression vectors, DNA sequences for gRNAs were synthesized and cloned into PUC19 or pLKO.1-TRC containing direct repeats of each Cas 13 correspondingly. For construction of shRNA expression vectors, DNA sequences of shRNAs were synthesized and individually cloned into the pLKO.1-TRC vector. DNA sequences of all gRNAs and shRNAs are listed in Supplementary Table 6.

Plasmid transfection was carried out using Lipofectamine 3000 Reagent (ThermoFisher) according to manufacturer's protocols. About $80 \%-90 \%$ transfection efficiency was achieved in HeLa and 293FT cells. Cells were harvested upon 48h after transfection for further RNA expression analysis.

\section{Lentivirus production and cell infection}

To produce lentiviral particles, $5 \times 10^{6} 293 \mathrm{FT}$ cells in a 10 -cm dish were co-transfected with $10 \mu \mathrm{g}$ expression vector of interest, $7.5 \mu \mathrm{g}$ psPAX2 vector and $3 \mu \mathrm{g}$ pMD2.G vector. The supernatant containing viral particles was harvested upon $48 \mathrm{~h}$ and $72 \mathrm{~h}$ after transfection and passed through Millex-GP Filter Unit ( $0.22 \mathrm{~mm}$ pore size, Millipore). Viral particles were then concentrated 100 -fold by sucrose gradient ultracentrifugation, resuspended in PBS containing $0.1 \% \mathrm{BSA}$, and stored at $-80^{\circ} \mathrm{C}$ till use. To infect HeLa or $293 \mathrm{FT}$ cells with lentivirus, cells were incubated with culture medium containing 10 $\mu \mathrm{L}$ concentrated lentivirus and $5 \mathrm{mg} / \mathrm{mL}$ polybrene (Sigma). Infected cells were treated with puromycin for several days to increase knockdown efficiency.

\section{Generation of stable cell lines with RfxCas13d expression}

p23-RfxCas13d-mcherry-flag vector was infected into HeLa or 293FT cells by lentivirus for stable cell line generation. Single clones with red fluorescence were selected and validated by WB using anti-Flag antibodies.

\section{RNA isolation and $q R T-P C R$}

Total RNAs from cultured cells were extracted with Trizol (Life Technologies) according to the manufacturer's protocol. RNAs were treated with DNase I (Ambion, 
DNA-freeTM kit). cDNAs were reverse transcribed with PrimeScript RT Reagent Kit (Takara) according to the manufacturer's protocol for qPCR. ACTB was examined as an internal control for normalization. Primers for all qRT-PCR assays were listed in Supplementary Table 6 .

\section{Northern Blotting (NB)}

NB was performed according to the manufacturer's protocol (DIG Northern Starter Kit, Roche). Digoxigenin (Dig)-labeled antisense riboprobes were made using RiboMAX Large Scale RNA Production Systems (Promega). In brief, $5 \mu \mathrm{g}$ total RNAs were resolved on denaturing urea polyacrylamide gel, transferred to nylon membrane (Roche) and UV-crosslinked using standard manufacturer's protocols. Membrane was then hybridized with specific Dig-labeled riboprobes. Primers for NB probes to detect $\operatorname{circPOLR} 2 A$ were listed in Supplementary Table 6.

\section{Western Blotting (WB)}

Cells were harvested after treated and resuspended in lysis buffer $(150 \mathrm{mM} \mathrm{NaCl}, 1 \%$ NP-40, $0.5 \%$ sodium deoxycholate, $0.1 \%$ SDS, 50mM Tris, pH8.0, and protease inhibitors) for $10 \mathrm{~min}$. After centrifugation, supernatants of cell lysates were resolved on $10 \%$ SDS-polyacrylamide gel and analyzed by WB with anti-Flag (Sigma), antiFAM120A, anti-IGF2BP2, anti-IGF1BP1, anti-GAPDH or $\beta$-actin (Sigma) antibodies.

\section{Cell proliferation assays}

To validate the observed effect of a specific circRNA on cell proliferation, BSJ-gRNAs targeting BSJ site of the circRNA were individually infected into HeLa or 293FT cell lines stably expressing RfxCas13d. Cells with specific circRNA-knockdown were obtained by puromycin selection for several days. Knockdown efficiencies of circRNAs by RfxCas13d/BSJ-gRNAs or shRNAs were measured prior to performing cell proliferation assays.

Cell proliferation was detected by an MTT assay that measures $\mathrm{OD}_{490}$ according to CellTiter 96 AQueous One Solution Cell Proliferation Assay (Promega), or by Ensight ${ }^{\circledR}$ Multimode Plate Reader (PerkinElmer) that measures the occupied surface area of cells to indicate confluency. $5 \times 10^{3} \mathrm{HeLa}$ or 293FT cells were seeded into each well of $96-w e l l$ dishes, then cultured at $37^{\circ} \mathrm{C}$ in a $5 \% \mathrm{CO}_{2}$ cell culture incubator for indicated time points. Cell proliferation at Day 0 was detected after cells were seeded for $5 \mathrm{~h}$ to allow their attachments to the plate. Live cells at D1, D2 and D3 were detected by MTT or by Ensight ${ }^{\circledR}$ Multimode Plate Reader. Data were normalized to the value of Day 0.

\section{Subcutaneous xenograft tumor Models}

All animal procedures were performed under the ethical guidelines of the Shanghai Institute of Biochemistry and Cell Biology, Chinese Academy of Sciences, CAS Center for Excellence in Molecular Cell Science. For subcutaneous xenograft tumor formation assay, 4 to 5-week-old male BALB/c-nu/nu mice (five mice per group) were injected subcutaneously with $1 \times 10^{6}$ cells. When mice were sacrificed, the tumors were collected and weighed.

\section{Subcellular fractionation}

Nuclear and cytoplasmic fractionation was carried out as described ${ }^{38}$ with slight modifications. Briefly, $3 \times 10^{6}$ cells were washed with PBS and collected by 
centrifugation at $1,000 \mathrm{rpm}$ for $2 \mathrm{~min}$ at room temperature. Cells were resuspended in $300 \mu \mathrm{L}$ lysis buffer (DPBS containing 1\% NP-40, 0.5\% sodium deoxycholate, $5 \mathrm{mM}$ EDTA, 1mM DTT, 1mM PMSF, $2 \mathrm{mM}$ RVC, $15 \%$ glycerol, $1 \mathrm{x}$ proteinase inhibitor cocktail), incubated on ice for $5 \mathrm{~min}$ and then examined under an optical microscope to ensure that membranes of most of cells were disrupted. Cells were centrifuged at 4,000 $\mathrm{x} \mathrm{g}$ for $1 \mathrm{~min}$ at $4^{\circ} \mathrm{C}$ and the supernatant was saved as cytoplasmic fractionations. The precipitated nuclei were further washed once with lysis buffer, resuspended in $300 \mu \mathrm{L}$ lysis buffer, sonicated and centrifuged at $13,000 \mathrm{rpm}$ for $10 \mathrm{~min}$ at $4^{\circ} \mathrm{C}$. The supernatant was saved as nuclear extracts.

\section{In vitro $\mathrm{RNA}$ transcription, circularization and purification}

In vitro RNA transcription assay was carried out as previous described ${ }^{18}$ with slightly modification. $1 \mu \mathrm{g}$ PCR-amplified T7-DNA fragments were incubated with $2 \mu \mathrm{L}$ T7 RNA polymerase enzyme and $0.5 \mathrm{mM}$ dNTPs. $2 \mathrm{mM}$ GMP was supplemented in the reaction to produce 5'-monophosphate RNA for FAM120A circularization. The reaction was carried out for $2 \mathrm{hr}$ at $37^{\circ} \mathrm{C}$, followed by DNase I treatment for $30 \mathrm{~min}$ at $37^{\circ} \mathrm{C}$ to remove DNA templates. Transcribed RNAs were precipitated with ethanol, washed with $70 \%$ ethanol and resuspended in RNase-free water.

For FAM120A circularization, $50 \mu \mathrm{g}$ transcribed FAM120A were incubated with T4 RNA ligase 1 (NEB) in $500 \mu \mathrm{L}$ reaction for overnight at $16^{\circ} \mathrm{C}$ according to the manufacturer's protocol. Circularized FAM120A were treated with RNase $\mathrm{R}$ as described ${ }^{33}$. Circularized and linear FAM120A were precipitated with ethanol and resolved on denaturing urea polyacrylamide gel. Then visualized by Ethidium bromide staining. The corresponding to circular RNA band was excised and circular RNAs were purified.

\section{Circular/linear RNA pull-down}

Biotinylated linear and circular RNAs were heated for 5 min at $65^{\circ} \mathrm{C}$ in PA buffer $(10$ $\mathrm{mM}$ Tris $\mathrm{HCl} \mathrm{pH} \mathrm{7.5,} 10 \mathrm{mM} \mathrm{MgCl}_{2}, 100 \mathrm{mM} \mathrm{NH}_{4} \mathrm{Cl}$ ) and slowly cooled down to room temperature. Then, $5 \times 10^{6}$ cells were resuspended with $1 \mathrm{~mL}$ binding buffer $(10 \mathrm{mM}$ HEPES pH 7.0, $50 \mathrm{mM} \mathrm{KCl,} \mathrm{10 \%} \mathrm{glycerol,} 1 \mathrm{mM}$ EDTA, $1 \mathrm{mM}$ DTT, $0.5 \%$ Triton X100 , heparin $0.3 \mathrm{mg} / \mathrm{mL}$ ), sonicated and centrifuged at $13,000 \mathrm{rpm}$ for $10 \mathrm{~min}$ at $4{ }^{\circ} \mathrm{C}$. The supernatant was pre-cleared with Streptavidin Dynabeats (Invitrogen) for $30 \mathrm{~min}$ at room temperature, followed by incubation with folded RNAs for $30 \mathrm{~min}$ and with beads for $10 \mathrm{~min}$. Retrieved proteins were subjected to WB.

\section{Circular/ linear RNAs competition assay}

Synthesized linear and circular FAM120A were heated for $5 \mathrm{~min}$ at $65^{\circ} \mathrm{C}$ in RNAfolding buffer $(10 \mathrm{mM}$ HEPES and $10 \mathrm{mM} \mathrm{MgCl} 2)$ and slowly cooled down to room temperature. $0.5 \mu \mathrm{g}$ folded linear FAM120A and $0.5 \mu \mathrm{g} \operatorname{circFAM120A}$ were added to incubate with $1 \mu \mathrm{g}$ His-tagged IGF2BP2 for $2 \mathrm{hr}$ at $4^{\circ} \mathrm{C}$ in $0.2 \mathrm{~mL}$ binding buffer $(50$ mM HEPES at $\mathrm{pH} 7.0,150 \mathrm{mM} \mathrm{NaCl}, 10 \mathrm{mM} \mathrm{MgCl}_{2}, 0.1 \mathrm{mM}$ DTT, $0.5 \mathrm{mM}$ PMSF, $2 \mathrm{mM}$ RVC). circFAM120A or linear FAM120A binding with His-tagged IGF2BP2 were collected by anti-His antibody and extracted with Trizol (Life technologies) according to the manufacture's protocol. Northern blot used to test the relative binding abundance of circFAM120A or linear FAM120A.

\section{RNA Immunoprecipitation (RIP)}

Cells growing in $15 \mathrm{~cm}$ dishes were rinsed twice with ice-cold PBS, harvested in 10 $\mathrm{mL}$ ice-cold PBS and then centrifuged at $1,000 \mathrm{rpm}$ for $5 \mathrm{~min}$ at $4^{\circ} \mathrm{C}$. Cell were 
resuspended in $1 \mathrm{~mL}$ RIP buffer (50 mM Tris, pH 7.4, $150 \mathrm{mM} \mathrm{NaCl}, 0.5 \%$ Igepal, $1 \mathrm{mM}$ PMSF, $1 \times$ protease inhibitor cocktail (Roche) and $2 \mathrm{mM} \mathrm{VRC)} \mathrm{and} \mathrm{subjected} \mathrm{to}$ three rounds of gentle sonication. Cell lysates were centrifuged at 12,000 rpm for 15 min at $4^{\circ} \mathrm{C}$ and the supernatants were precleared with $15 \mathrm{~mL}$ Dynabeads Protein $\mathrm{G}$ (Invitrogen) to get rid of non-specific binding. Then, the precleared lysates were used for IP with anti-AGO2 or anti-IGF2BP2 antibodies. IP was carried out for $2 \mathrm{hr}$ at $4^{\circ} \mathrm{C}$. The beads were washed three times with high salt buffer and two times with the RIP buffer, followed by extraction with elution buffer (100mM Tris, $\mathrm{pH} 6.8,4 \%$ SDS, and $10 \mathrm{mM}$ EDTA) at room temperature for $10 \mathrm{~min}$. One-third of the eluted sample was used for WB and the remaining was used for RNA extraction. The RNA enrichment was assessed by qRT-PCR. Primers are listed in Supplementary Table 6.

\section{Ribosome fractionation}

circFAM120A KD and control cells were exposed to cycloheximide $(100 \mu \mathrm{g} / \mathrm{mL})$ for $15 \mathrm{~min}$, then $2 \times 10^{7}$ cells were lysed in $500 \mu \mathrm{L}$ lysis buffer $(20 \mathrm{mM}$ Tris $\mathrm{pH} 7.4,15$ $\mathrm{mM} \mathrm{MgCl} 2,200 \mathrm{mM} \mathrm{KCl}, 1 \%$ Triton X-100, $100 \mu \mathrm{g} / \mathrm{mL}$ cycloheximide, $1 \mathrm{mM}$ DTT, 1 $\mathrm{mg} / \mathrm{mL}$ heparin and $40 \mathrm{U} / \mathrm{mL}$ RNasin (Promege)), and centrifuged at $13,000 \mathrm{rpm}$ at $4{ }^{\circ} \mathrm{C}$ for $10 \mathrm{~min}$. For fractionation, the lysates were loaded on $10 \%-45 \%$ sucrose gradients and sequenced by ultracentrifugation with a SW41 rotor (Beckman) at 36,000 rpm at $4^{\circ} \mathrm{C}$ for $2.5 \mathrm{hr}$. Linear sucrose gradients were prepared with a Gradient Master (Biocomp) as indicated by the manufacturer. The distribution of ribosomes on the gradients was recorded at $254 \mathrm{~nm}$ by BIOCOMP Piston Gradient Fractinator equipped with BIO-RAD ECONO UV Monitor.

\section{Zygote injection and in vitro embryo culture}

Around 8-week old B6D2F1 (C57BL/6 X DBA/2) female mice were superovulated and mated with the male B6D2F1 mice. 21-23 hr later, fertilized embryos were collected from oviducts. Afterwards, Rfx-Cas13d mRNAs (100 ng/ $\mu \mathrm{l})$ and gRNA (100 ng/ $\mu \mathrm{l})$ were mixed and injected into the cytoplasm of fertilized eggs in a droplet of HEPESCZB medium containing $5 \mu \mathrm{g} / \mathrm{ml}$ cytochalasin B (CB) using a FemtoJet microinjector (Eppendorf) with constant flow settings. The injected embryos were cultured in KSOM with amino acids at $37^{\circ} \mathrm{C}$ under $5 \% \mathrm{CO} 2$ in air until hatching blastocyst stage by 4 days.

\section{Candidate circRNA selection and gRNA design for RfxCas13d/BSJ-gRNA library}

For library of 762 circRNAs construction, we applied CIRCexplore $2^{19}$ to identify circRNAs in multiple cell lines (HT29, 293FT, HeLa and H9 cells). In total, 11,796 circRNAs were identified. To obtain highly expressed circRNAs, we selected the top $10 \%$ highly expressed circRNAs $(1,490)$ that present in at least one line of HT29, $\mathrm{HeLa}, 293 \mathrm{FT}$ or $\mathrm{H} 9$ cells. According to the reported 6 copies of $\operatorname{circPOLR} 2 A$ per HeLa cell ${ }^{18}$, we calculated $\operatorname{circPOLR} 2 A$ and other circRNA copy numbers in each cell line by the FPBcirc value of each circRNA from RNA-seq. In total, 1,179 circRNAs were selected by expression at $\geq 3$ copies in at least one cell line. Among them, 762 circRNAs (65\%) were successfully designed with BSJ-gRNAs and included in the gRNA library (Supplementary Table 1).

For each 762 circRNA, we designed five BSJ-gRNAs that all target sequences spanning BSJ with different lengths $(15 \mathrm{nt}+15 \mathrm{nt}$, 20nt $+10 \mathrm{nt}, 10 \mathrm{nt}+20 \mathrm{nt}, 14 \mathrm{nt}+$ $14 \mathrm{nt}$ and $13 \mathrm{nt}+13 \mathrm{nt}$ ). In addition, one paired control gRNA (random $15 \mathrm{nt}+$ matched $15 \mathrm{nt}$ ), in which their half-sequences were replaced by scrambled sequences, was designed for each circRNA and was included in the gRNA library (Supplementary Table 1). 
For library of 2,908 circRNAs construction, we applied CIRCexplore $2^{19}$ to identify circRNAs in multiple cell lines (HT29, 293FT, HeLa, H9, HepG2, K562, PA1, SHSY5Y and FB cells). In total, 50,911 circRNAs were identified. To obtain highly expressed circRNAs, we selected the top 800 highly expressed circRNAs $(3,177)$ of each cell lines. Among them, 2,908 circRNAs were successfully designed with BSJgRNAs and included in the gRNA library (Supplementary Table 2).

For each 2,908 circRNAs, we designed three BSJ-gRNAs that all target sequences spanning BSJ with different lengths $(15 \mathrm{nt}+15 \mathrm{nt}$, 20nt $+10 \mathrm{nt}$ and $10 \mathrm{nt}+20 \mathrm{nt})$. In addition, one paired control gRNA (random 15nt + matched 15nt), in which their halfsequences were replaced by scrambled sequences, was designed for each circRNA and was included in the gRNA library (Supplementary Table 2).

\section{Construction of a RfxCas13d/BSJ-gRNA library}

We created a library targeting 762 circRNAs and 2,908 circRNAs with 4,572 gRNAs and 11,832 gRNAs as mentioned above. Oligonucleotides containing gRNA sequences were synthesized in Genscript company. Then, primers matching flanking sequences of oligonucleotides were used for the amplification to create 60-bp homologies with BsmBI digested gRNA-expressing backbone ${ }^{39}$. The amplified DNA products were assembled into the BsmBI digested gRNA-expressing backbone using Gibson assembly method and were transformed into E. coli DH5a Electro-Cells (Takara) by electroporation to obtain the library plasmids. The lentivirus of the gRNA library was produced by co-transfection of library plasmids with psPAX2 and pMD2.G plasmids into 293FT cells using the Lipofectamine 3000 Reagent.

\section{Screening for circRNAs with functional potential using the RfxCas13d/gRNA library}

To screen circRNAs that are important for cell proliferation, RfxCas $13 \mathrm{~d}$ stable cells were infected with the gRNA lentiviral library at a multiplicity of infection (MOI) of 0.3 . After selection by puromycin for one day, $2 \times 10^{6}$ infected cells were harvested as D1 samples. And an aliquot of $2 \times 10^{6}$ infected cells were cultured and passaged for 30 days as D30 samples. Two biologically independent experiments were performed both in HeLa and 293FT cells. All samples were used for DNA extraction, gRNA enrichment by PCR amplification and deep sequencing.

To screen circRNAs whose loss may confer resistance of cells to doxorubicin (DOX) treatment, RfxCas13d stable 293FT cells were infected with the gRNA lentiviral library at a MOI of 0.3 . After selection by puromycin for one day, $2 \times 10^{6}$ infected 293FT cells were cultured and passaged for 7 days. Then infected cells were treated with DMSO or DOX $(1 \mu \mathrm{M})$ for 2 days, survived cells were collected and used for DNA extraction, gRNA enrichment by PCR amplification and deep sequencing. Two biologically independent experiments were performed.

Deep sequencing of amplified PCR products containing gRNA sequences was conducted by Illumina NextSeq 500 at CAS-MPG Partner Institute for Computational Biology Omics Core, Shanghai, China.

\section{Computational analysis of circRNA screens}

Adapters at 3 ' end and 5 ' end in the raw paired-end sequencing datasets were removed by cutadapt (1.16) using the following adapter sequences: 


\section{R1: -a TTTTTTAAGCTTGGCGTAACTAGATCT -m 15, -g CCCTACCAACTGGTCGGGGTTTGAAAC -m 15; R2: -a GTTTCAAACCCCGACCAGTTGGTAGGG -m 15, -g AGATCTAGTTACGCCAAGCTTAAAAAA -m 15.}

Processed reads were aligned to gRNA library sequences with Bowtie (v1.1.2, parameters: -v $3-\mathrm{m} 1-\mathrm{k} 1)$. Only uniquely mapped reads of each sample were calculated.

The read number for each unique gRNA was normalized as previously reported ${ }^{40}$. Counts of gRNAs from two biological replicates were assessed by Pearson correlation coefficient (PCC). Average of normalized reads from two biological replicates were used as the normalized read count for each gRNA.

RRA score of each circRNA was calculated by MAGeCK that evaluates the statistical significance of individual gRNA abundance change using a negative binomial model ${ }^{20}$. CircRNAs were ranked by RRA scores with a null model of a uniform distribution. A small RRA score indicates a strong selection of circRNAs during the screening. Fifty circRNAs with the lowest RRA scores were selected, and their normalized gRNA reads of paired-controls were unchanged between D1 and D30 samples. Finally, circRNAs with top 50 RRA score in HT29, 293FT or HeLa cells were selected as potential candidates. See Extended Data Fig. 5.

\section{Polyadenylated RNA library preparation, deep sequencing and analysis}

Polyadenylated [poly(A)+] RNA enrichment was performed as described ${ }^{41}$. Poly(A)+ RNA-seq libraries were prepared using Illumina TruSeq Stranded mRNA Sample Prep Kits and subjected to deep sequencing with Illumina NextSeq 500 at the CAS-MPG Partner Institute for Computational Biology Omics Core, Shanghai, China. RNA-seq sequencing read quality was evaluated by FastQC (v0.11.5).

Deep sequencing datasets were mapped with TopHat (TopHat v2.0.12, parameters: microexon-search -g 1 -a 6 -m 2) and aligned to GRCh37/hg19 human reference genome with the UCSC Genes annotation (Human: hg19 knownGene.txt updated at 2013/06/30). Gene expression of linear mRNAs was determined by FPKM (Fragments Per Kilobase of transcript per Million mapped fragments). The maximum FPKM of expressed transcript was selected to represent the expression level of each given gene. Refseq genes with FPKM $\geq 1$ at least in one sample were selected for comparison (Human hg19 refFlat.txt updated at 2017/04/09), and mean of two replicates was calculated as the gene expression level. Fold change (FC) between gRNA (or shRNA) $\mathrm{KD}$ and RfxCas13d NT (or shRNA NT) was used to determine upregulated (FC $\geq 1.5$ ), unchanged $(0.667<\mathrm{FC}<1.5)$ and downregulated $(\mathrm{FC} \leq 0.667)$ genes.

\section{Gene Ontology (GO) of Differentially Expressed Genes (DEGs)}

DEGs after circFAM120A knockdown by RfxCas13d gRNA were identified for GO. Ninety seven DEGs were manually clustered with https://www.genecards.org/ (GeneCards) and https://www.ncbi.nlm.nih.gov/pubmed/ (PubMed). Annotated GO terms (http://amigo.geneontology.org/amigo) were employed to classify circFAM120Aaffected genes based on their functions with a manual check $^{42}$.

\section{Conservation of circRNAs analyses}


Conservation of circRNAs analysis pipeline according to previous publications ${ }^{35}$ with some modification. In brief, sequences of back-spliced exons were extracted and used LiftOver tool (http://genome.ucsc.edu/cgi-bin/hgLiftOver, parameters: -bedPlus D 3 -tab -minMatch D 0.1 -minBlocks D 1) to identify orthologous coordinates between human (genome version: hg19) and mouse (genome version: mm10). In addition, an expressed circRNA identified in mouse orthologous locus without nucleotide difference and also expressed in mouse was suggested as a conserved circRNA. Similar strategy was also applied to mouse circRNAs.

\section{Statistical analyses}

$P$ values $<0.05,<0.01$ and $<0.001$ were marked by 1 asterisk, 2 asterisks or 3 asterisks, respectively. Statistically significant difference was assessed using Wilcoxon test with R platform (R v.3.5.1) and statistical significance was set at $P<0.05$. To evaluate the relevant correlation between two datasets, Pearson correlation coefficient (PCC) was performed with $\mathrm{R}$ platform (R v.3.5.1). Statistically significant difference for cell proliferation was assessed using $\mathrm{T}$ test, and statistical significance was set at $P<0.05$.

\section{Data availability}

All sequencing datasets have been deposited in the NCBI GEO (GSEXXX) and National Omics Data Encyclopedia (OEPxxxxxx). 


\section{Supplemental Figures and Figure Legends}

a

c
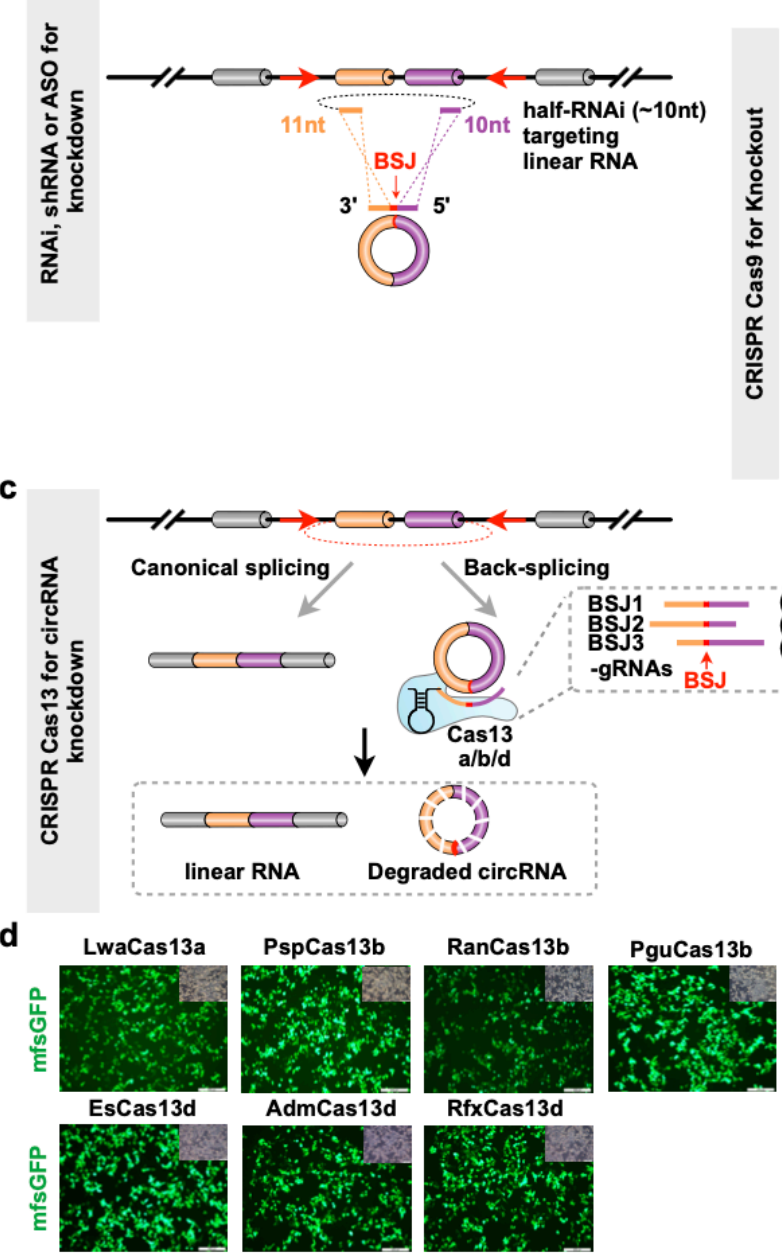

f

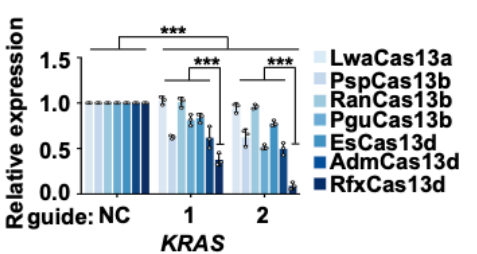

g : $: \mathrm{RT}_{\mathrm{R}} \mathrm{Cas} 13 \mathrm{~d}$

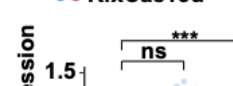

喜 $1 . 5 \longdiv { n s }$

Other Cas13 a/b/d

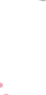
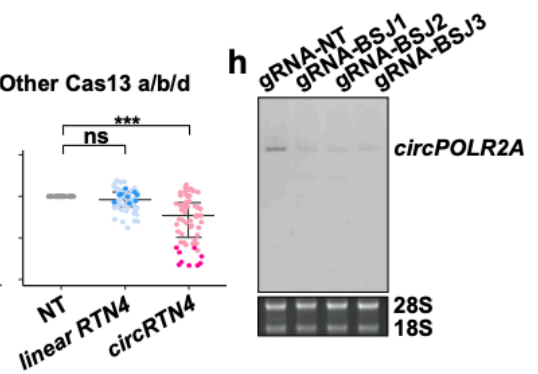

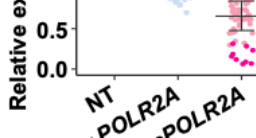

$a^{3 x^{p}}$ i

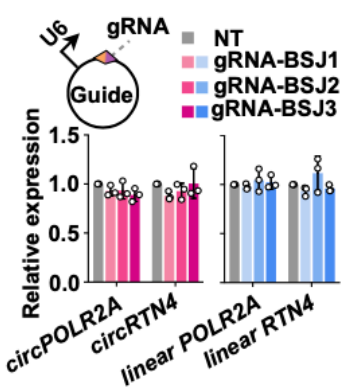

j

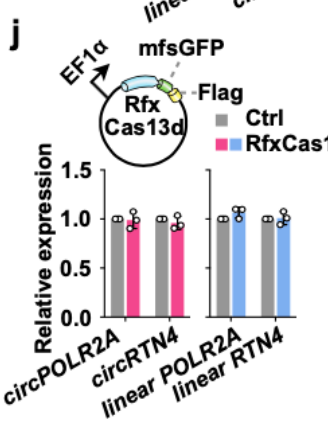

CircRNA-forming exon deletion

Intronic RNA pair disruption:
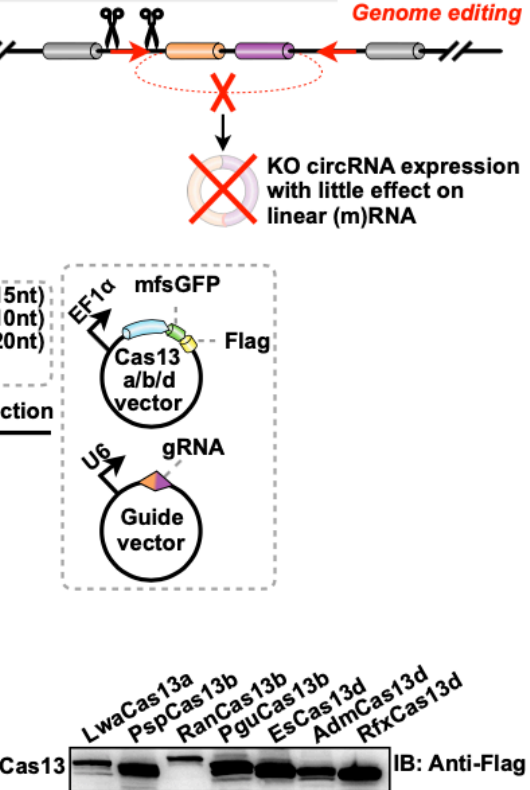

АСтв

$----$ k

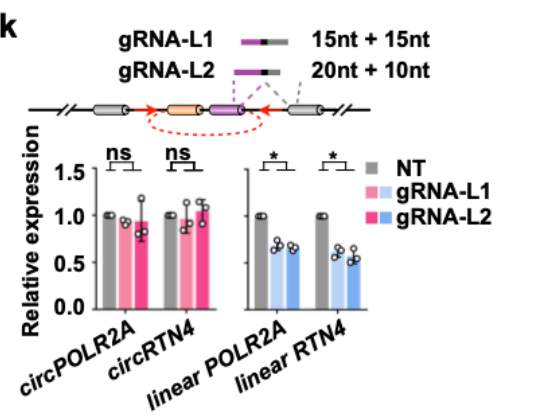

Extended Data Fig. 1 | Currently applied interference technologies for circRNA expression and evaluation of RfxCas13d knockdown efficiency and specificity.

a, Strategy of circRNA knockdown by RNAi machinery, such as RNAi, shRNA and ASO. The BSJ is a featured site for specific targeting of circRNA. Note, even halfRNAi ( 10nt) can target the linear cognate RNA. 
b, Strategies of circRNA knockout by CRISPR/Cas9. Top, genome editing is used to directly remove the circRNA-formed exons; bottom, genome editing destroys the intronic RNA pair to block circRNA expression with little effect on the linear cognate RNA.

c, Schematic of circRNA knockdown by Cas13 orthologues. Cas13a/b/d sequences were individually cloned into the vector with sequences for a $\mathrm{C}$-terminal monomeric superfolder GFP (msfGFP) and a Flag-tag. Three guide RNAs targeting the backsplicing junction site (BSJ, red arrow) were designed for each circRNA (BSJ-gRNAs). Expression levels of circRNAs and their cognate linear mRNAs were detected by qRTPCR. NT, non-targeting guide RNA.

d,e, Expression levels of Cas 13 proteins were detected by msfGFP fluorescence (d) and western blot (e) in 293FT cells after $48 \mathrm{~h}$ transfection.

f, Comparison of different Cas 13 proteins-mediated $K R A S$ knockdown efficiencies with two position-matched guides revealed that RfxCas $13 \mathrm{~d}$ is the best effector ${ }^{15}$.

g, Evaluation of different Cas13-protein-mediated knockdown on circRNAs. Expression levels of two circRNAs, $\operatorname{circPOLR2A}$ and $\operatorname{circRTN4}$, and their cognate mRNAs were detected by qRT-PCR in 293FT cells. NT, non-targeting guide RNA.

h, Northern blot confirmed the $\operatorname{circPOLR} 2 A$ knockdown by RfxCas13d/BSJ-gRNAs. $\mathbf{i}, \mathbf{j}$, Overexpression of individual BSJ gRNAs (i) or RfxCas13d (j) alone did not affect $\operatorname{circPOLR} 2 A$ and $\operatorname{circRTN} 4$ expression.

$\mathbf{k}$, gRNAs with partial sequences replaced by adjacent linear exon led to linear but not circular RNA knockdown. Top, schematic design of gRNAs that target exons with partial sequences overlapped with circRNAs, and the remaining sequences overlapped with their linear mRNA exons (gRNA-L). Bottom, knockdown efficiencies of circPOLR2A and circRTN4 as well as their corresponding linear RNAs under the treatment of each control gRNA-L and RfxCas $13 \mathrm{~d}$ were detected by qRT-PCR.

All transcript levels were normalized to $A C T B$, and values represent mean $+/-\mathrm{SD}$ with $\mathrm{n}=3, *: P<0.05 ; * *: P<0.01 ; * * *: P<0.001$; ns, not significant. 
a
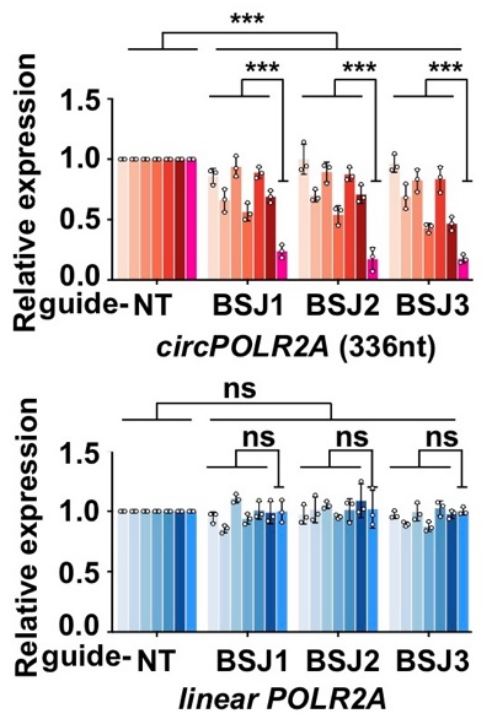
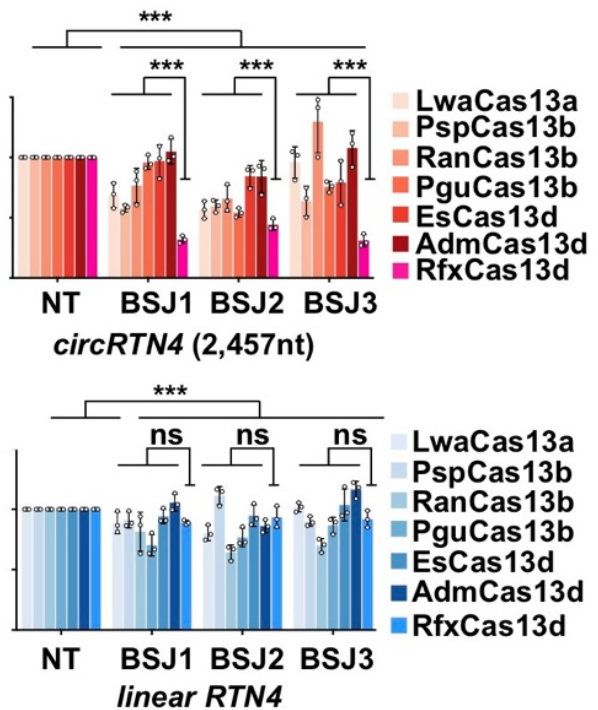

b
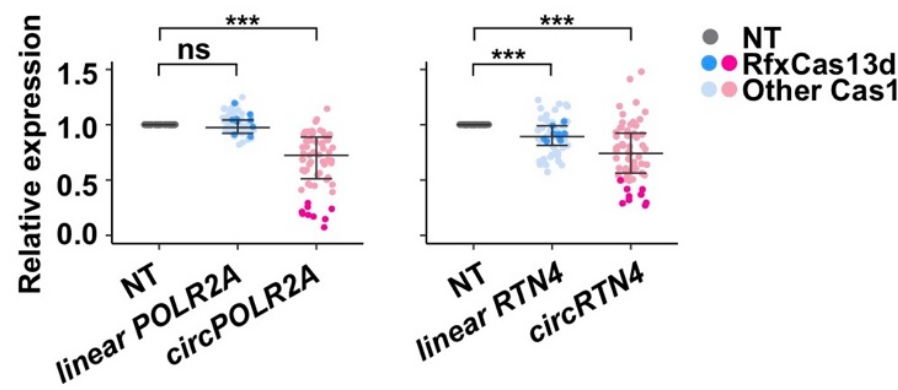

Other Cas13 a/b/d

C

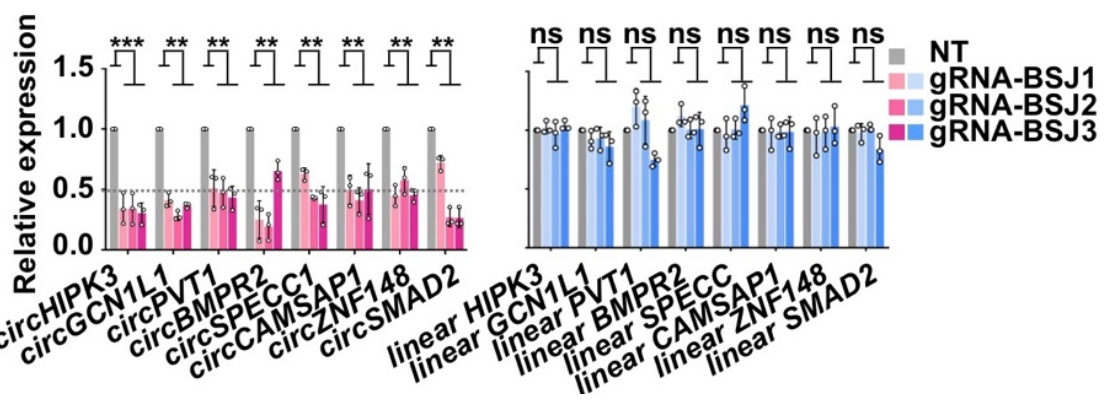

Extended Data Fig. 2 | RfxCas13d is the best effector to mediate circRNA knockdown in HeLa cells.

a,b, Evaluation of different Cas13 proteins mediated knockdown on circRNAs (circPOLR2A and circRTN4) and their cognate mRNAs in HeLa cells revealed that RfxCas13d is the best effector for the circRNA-specific knockdown.

c, The RfxCas13d/BSJ-gRNA system mediates specific and robust knockdown of randomly selected circRNAs, but not their cognate mRNAs in HeLa cells.

All transcript levels were relative to $A C T B$, and values represent mean $+/-\mathrm{SD}$ with $\mathrm{n}=3$, **: $P<0.01 ; * * *: P<0.001$; ns, not significant. 
a

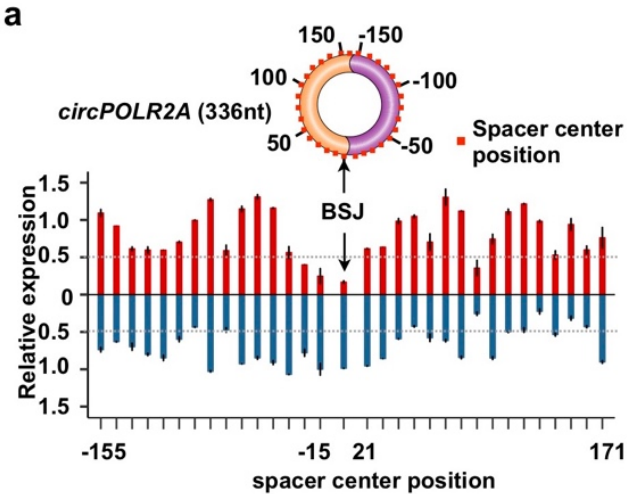

C

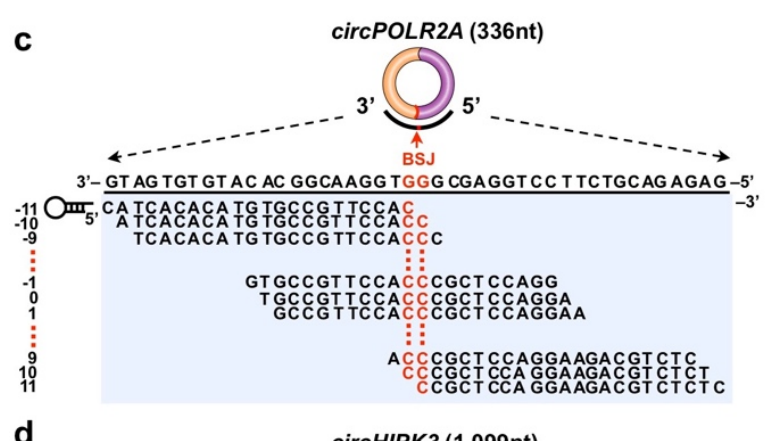

d

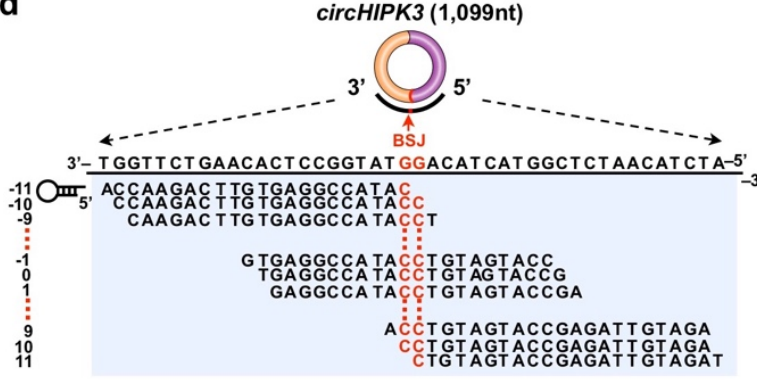

e

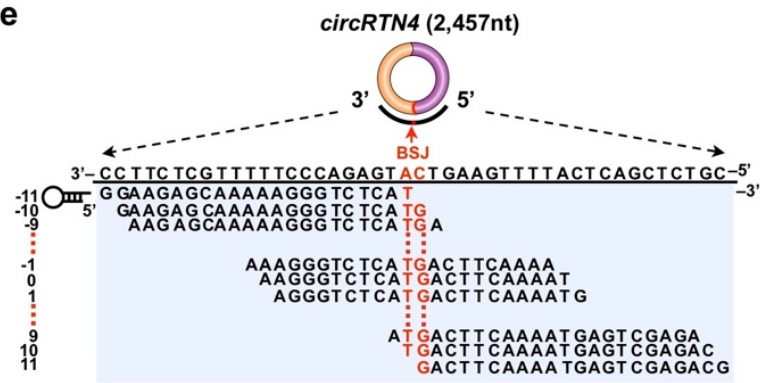

b
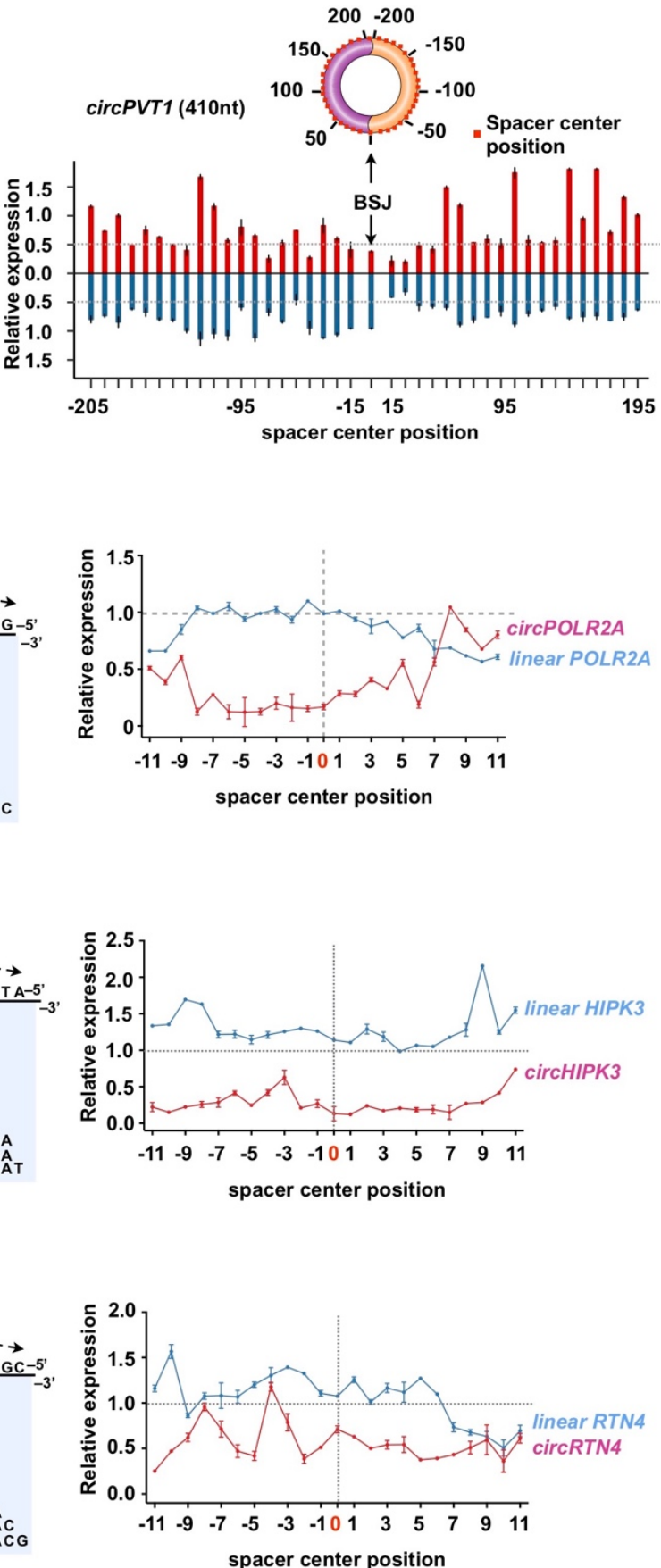

Extended Data Fig. 3 | RfxCas13d arrayed screening of BSJ site of targeted circRNAs.

$\mathbf{a}, \mathbf{b}$, Efficiency and specificity of RfxCas $13 \mathrm{~d}$ for circRNA and cognate linear mRNA knockdown. Top, schematic of gRNAs targeting $\operatorname{circPOLR} 2 A$ (a) and $\operatorname{circPVT1}$ (b) tiled 10-nt increments away from the BSJ site. Bottom, knockdown efficiencies of circRNA and linear RNA by each gRNA and RfxCas13d were detected by qRT-PCR. c-e, Arrayed knockdown screen of 23 guides evenly tilled across BSJ of $\operatorname{circPOLR} 2 \mathrm{~A}$ (c), circHIPK3 (d) and circRTN4 (e). Position-effect of BSJ-gRNAs for RfxCas13dmediated knockdown of $\operatorname{circPOLR} 2 A$ (c), $\operatorname{circHIPK} 3$ (d) and $\operatorname{circRTN4}$ (e) at the single nucleotide level. Twenty-three guides tiled across the BSJ of $\operatorname{circPOLR2A}$ (c), circHIPK3 (d) and circRTN4 (e) are listed. Knockdown efficiencies of circPOLR2A (c), circHIPK3 (d) or circRTN4 (e) and linear POLR2A, linear HIPK3 or linear RTN4 by each BSJ-gRNA were detected by qRT-PCR. 
All transcript levels are relative to $A C T B$, and values represent mean $+/-\mathrm{SD}$ with $\mathrm{n}=3$.

a

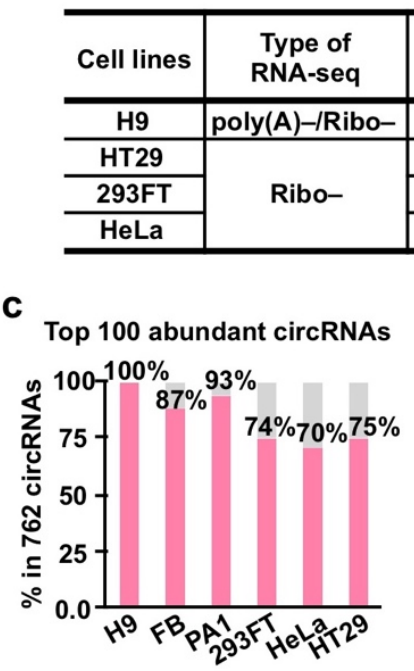

f

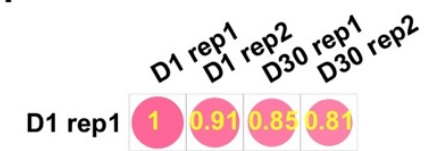

D1 rep2

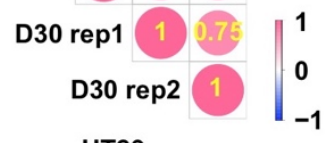

HT29

i

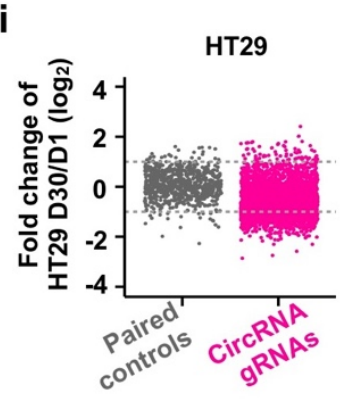

I

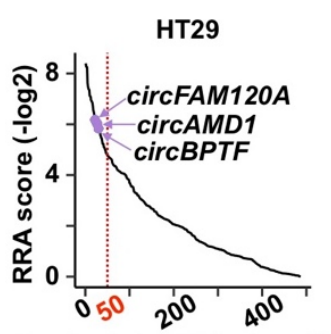

Rank of circRNAs $(n=487)$

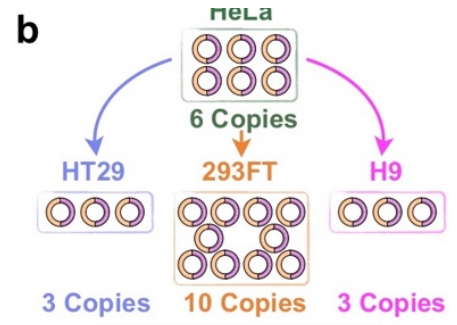

Copies $=$ FPBcirc $/ K^{*}$ d

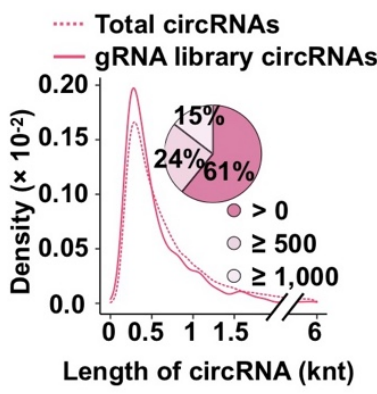

g

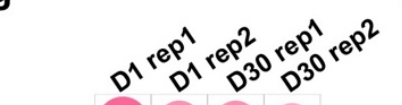

e

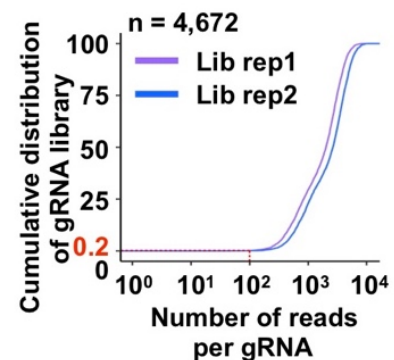

h

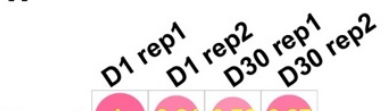

D1 rep1

D1 rep2

D1 rep2

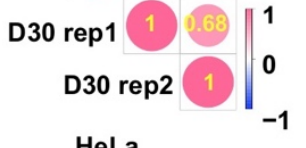

k

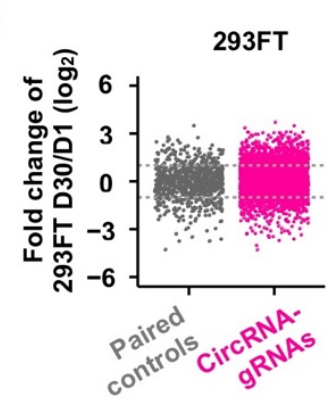

m

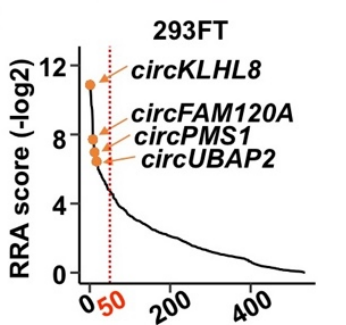

Rank of circRNAs $(n=535)$

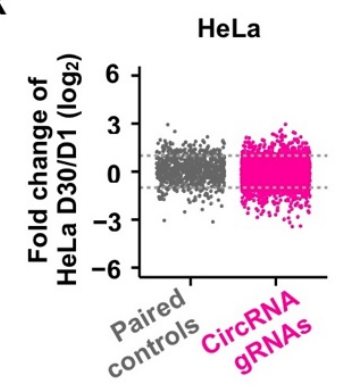

n

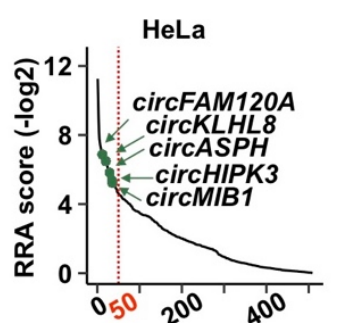

Rank of circRNAs $(n=509)$

Extended Data Fig. 4 | Characterization of the BSJ-gRNA library targeting 762 circRNAs and identification of negatively selected candidate circRNAs important for cell growth.

a, Calculation of circRNA copy numbers (using $\operatorname{circPOLR} 2 A$ as an example) in H9, HT29, HeLa and 293FT cells. According to the FPM of circPOLR2A and other circRNAs in H9, HT29, HeLa and 293FT cells, and the known six copies of $\operatorname{circPOLR} 2 A$ per HeLa cell ${ }^{18}$, we calculated the copy number of all circRNAs, 
respectively. For example, there are three copies of circPOLR2A per H9 or HT29 cells. b, Schematic of circRNA copy number calculation in H9, HT29, HeLa and 293FT cells. c, Representation of 762 candidate circRNAs designed with BSJ-gRNAs in the library in different cell lines. More than $70 \%$ of the top 100 abundant circRNAs in each indicated cell line were included in the 762 candidate circRNAs.

d, Matched length distribution of 762 candidate circRNAs (solid line) and total circRNAs (dashed line). Density curve and pie chart show that more than $80 \%$ of 762 candidate circRNAs and total circRNAs are less than 1,000nt.

e, Cumulative distribution of the number of reads per gRNA of constructed libraries. The red line indicates that less than $0.2 \%$ of gRNAs are covered by less than 100 reads. $\mathbf{f}-\mathbf{h}$, The Pearson correlation coefficient (PCC) between replicates (rep) of D1 and D30 samples in HT29 (f), 293FT (g) and HeLa cells (h). Two biologically independent experiments were performed at D1 and D30 in each cell line.

i-k, Scatter plot of fold change of paired controls and circRNA BSJ-gRNAs between D1 and D30 samples in HT29 (i), 293FT (j) and HeLa cells (k). The grey dashed lines indicate 2 or 0.5 fold change, respectively.

l-n, Rank of negatively selected candidate circRNAs by RRA scores in HT29 (l), 293FT (m) and HeLa cells (n). Fifty circRNAs with the lowest RRA scores were sub-grouped by red dashed line. 
a

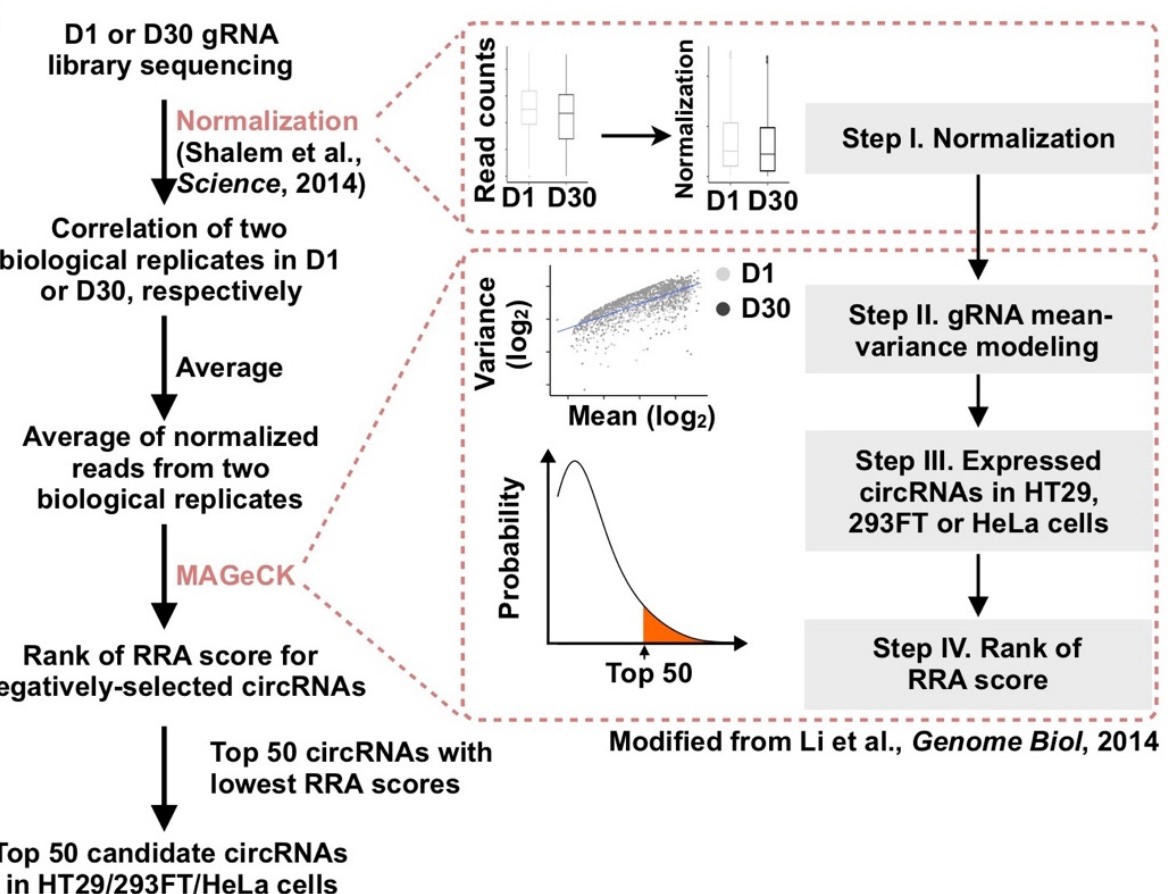

b

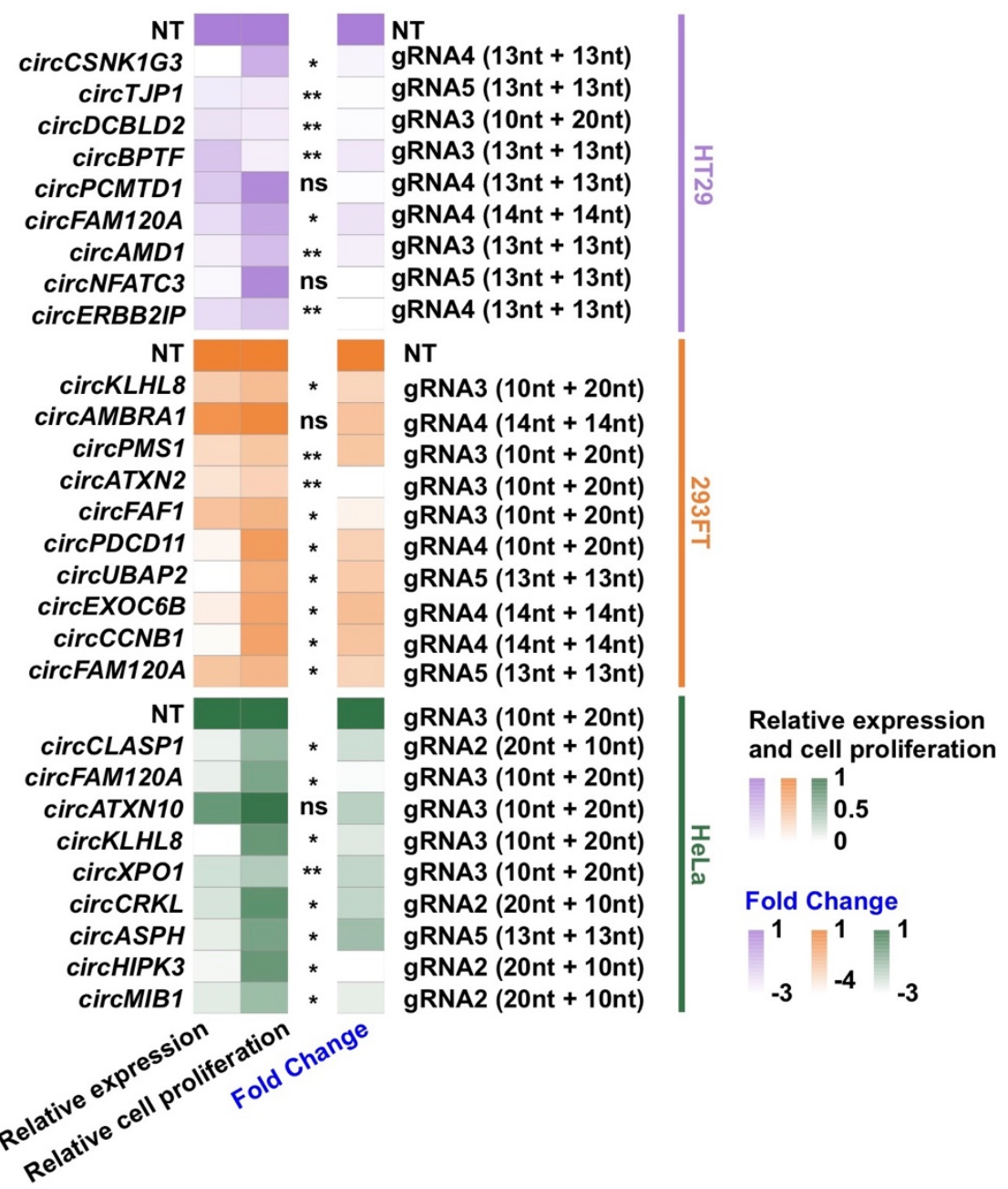

Extended Data Fig. 5 | Validation of 25 candidate circRNAs by the RfxCas13d system in cell proliferation

a, Schematic of circRNA candidates identification. Raw read counts were normalized 
according to previous publication ${ }^{40}$. Since two biological replicates are well correlated, the averaged read counts were used for the subsequent analysis. RRA scores were calculated by the MAGeCK algorithm to determine top negatively selected circRNAs with low RRA scores. After that, expressed circRNAs in HT29, 293FT and HeLa cells were selected for future analysis.

b, Heatmap display of the relative knockdown efficiency, cell proliferation and fold change of candidate circRNAs in HT29 (purple), 293FT (orange) and HeLa (green) cells with single BSJ-gRNA that targets each candidate circRNA. 
a

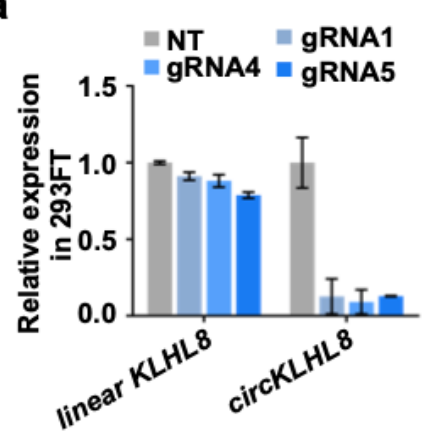

b

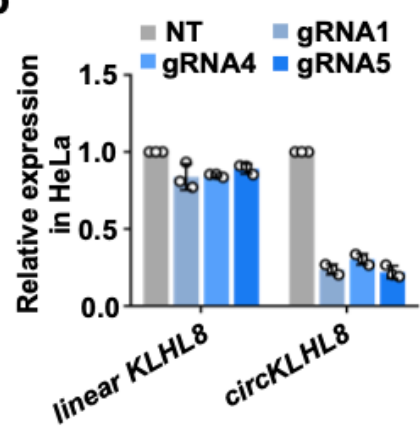

C

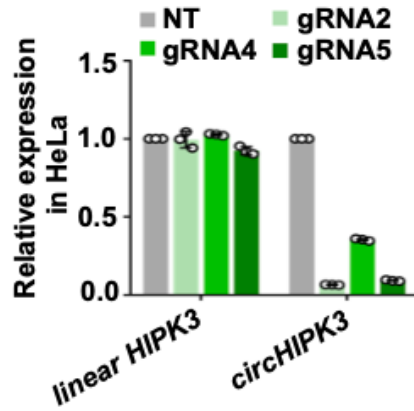

d

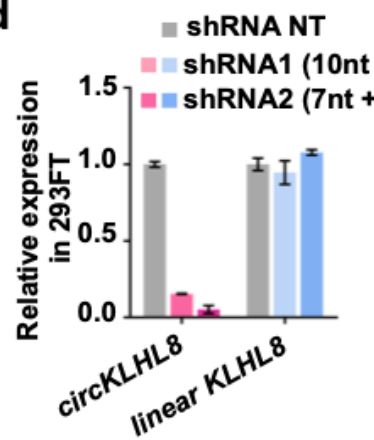

$+11 n t)$

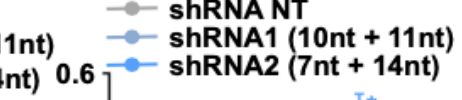

NT

- gRNA4 (14nt + 14nt)

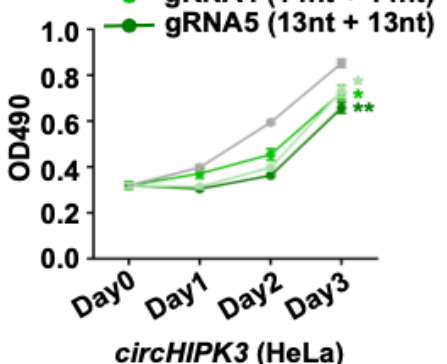

circHIPK3 (HeLa)

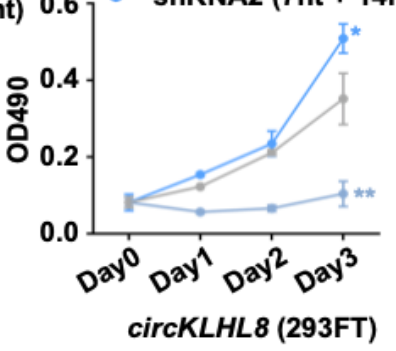

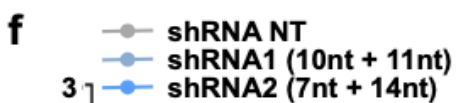

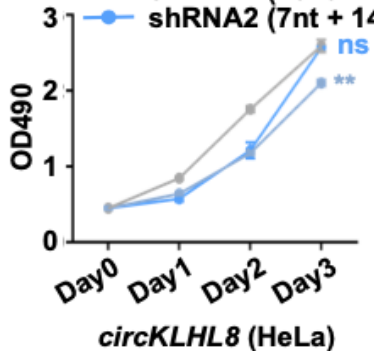

NT

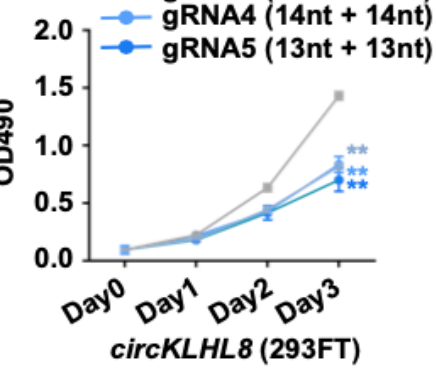

NT

gRNA1 (15nt + 15nt)
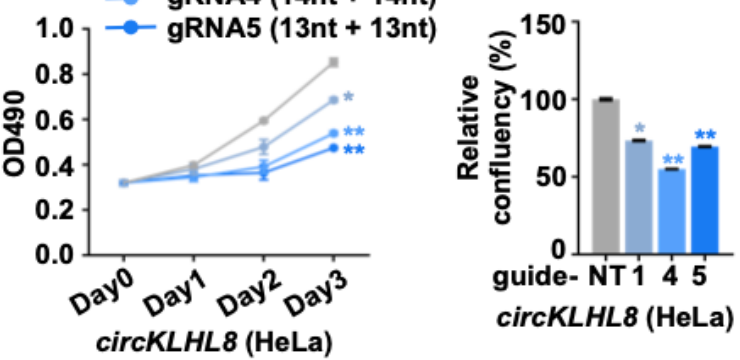

circKLHL8 (HeLa)

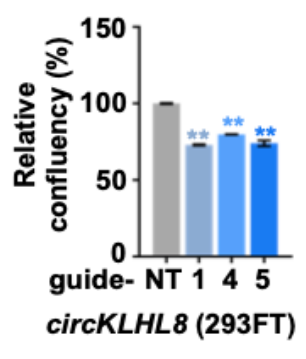

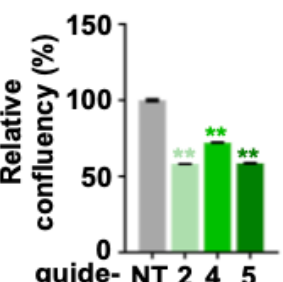

guide- NT 245

circHIPK3 (HeLa)

e

I ShRNA NT

ShRNA1 (10nt + 11nt)

m shRNA2 (7nt + 14nt)
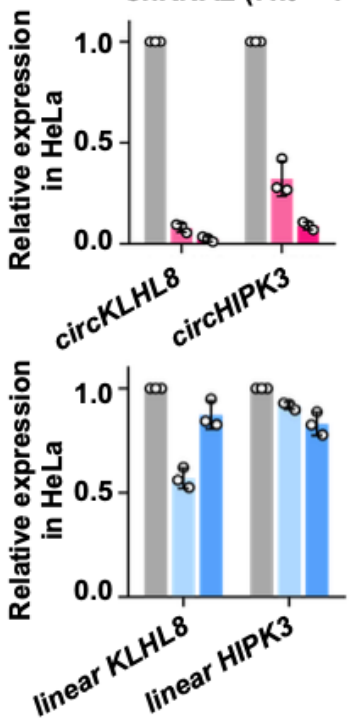

Extended Data Fig. 6 | Validation of $\operatorname{circKLHL8}$ and $\operatorname{circHIPK3}$ by the RfXCas13d 
system and shRNAs in cell proliferation.

$\mathbf{a}, \mathbf{b}$, Knockdown of circKLHL8 by RfxCas13d inhibited cell proliferation in 293FT (a) and HeLa cells (b), as revealed by MTT cell proliferation assay (Middle) and cell confluency calculated by the surface area occupied by cells (Right), the knockdown efficiency is showed in left.

c, Knockdown of circHIPK3 by RfxCas $13 \mathrm{~d}$ inhibited cell proliferation in HeLa cells, as revealed by MTT cell proliferation assay (Middle) and cell confluency calculated by the surface area occupied by cells (Right), the knockdown efficiency is showed in left. All transcript levels were normalized to $A C T B$ by qRT-PCR, and values represent mean $+/$ SD with $n=3$, values represent mean $+/$ - SD with $n=3, *: P<0.05$; **: $P<0.01$; ns, not significant.

d, Knockdown of $\operatorname{circKLHL} 8$ by shRNA inhibited cell proliferation in 293FT cells. The left is knockdown efficiency, and the right is cell proliferation.

e, Knockdown of circKLHL 8 and circHIPK3 by shRNA in HeLa cells.

f, Individual knockdown of circKLHL8 or circHIPK3 by shRNA inhibited cell proliferation in HeLa cells, as revealed by MTT cell proliferation assay.

All transcript levels were normalized to $A C T B$ by qRT-PCR, and values represent mean $+/$ - SD with $n=3, *: P<0.05$; **: $P<0.01$; ns, not significant.

a

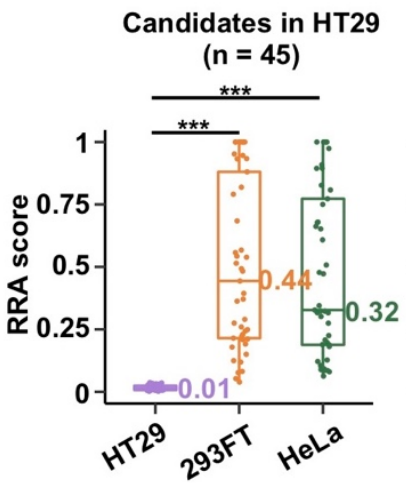

b

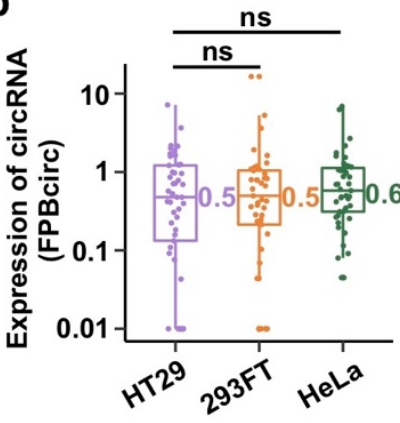

Candidates in 293FT $(n=43)$
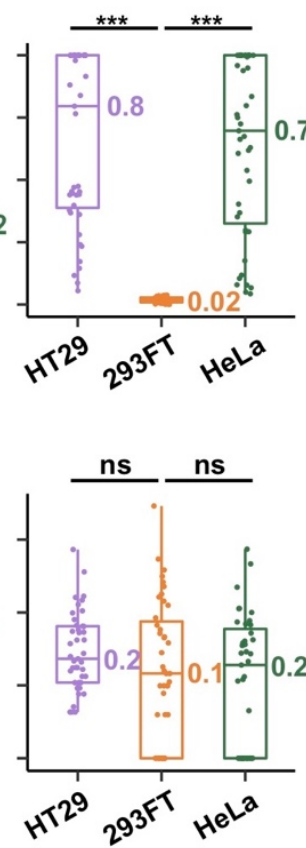

Candidates in HeLa $(n=41)$
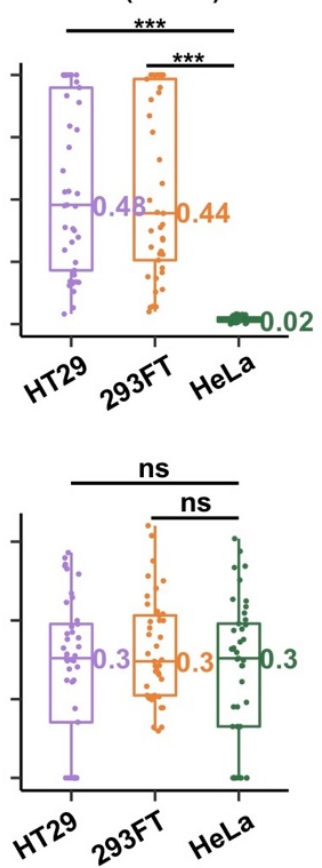

Extended Data Fig. 7 | Distribution of RRA score of and expression level of circRNAs that have cell type-specific effects on cell growth.

a, Distribution of the RRA score of circRNAs with cell-type specific effects in HT29 (45), 293FT (43) and HeLa (41) cell, respectively.

b. Distribution of expression (shown by FPBcirc) of circRNAs shown in (a) in HT29, 293FT and HeLa cells. 
a

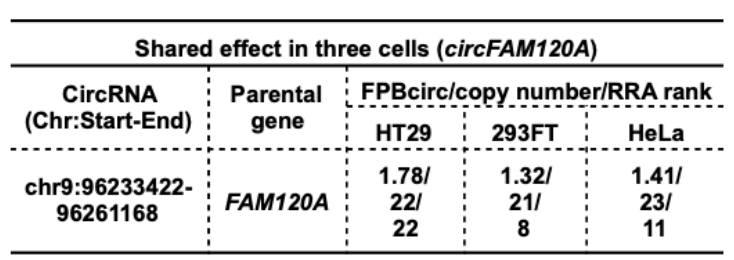

C
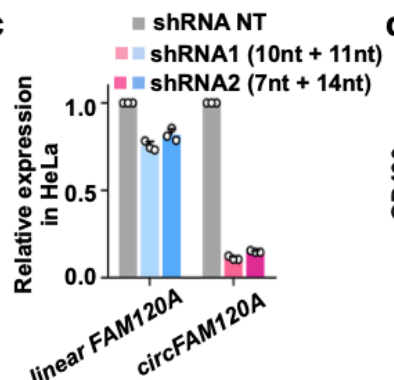

e

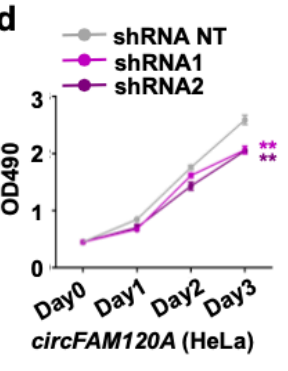

b

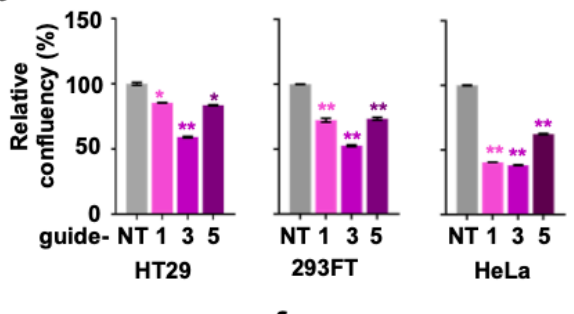

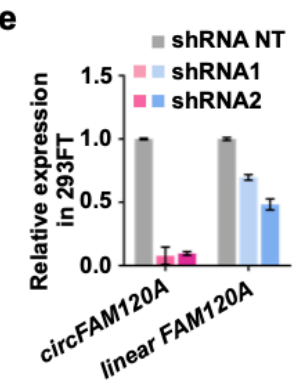

f

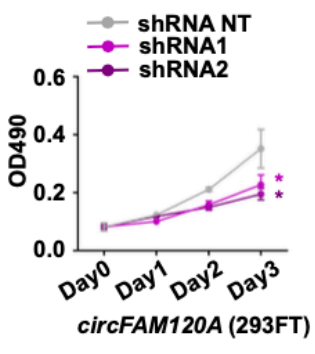

Extended Data Fig. 8 | circFAM120A promotes cell proliferation.

a, Summary of circFAM120A expression and growth defects in all three examined cell lines.

b, Knockdown of circFAM120A by RfxCas13d/BSJ-gRNAs inhibited HT29, 293FT or HeLa cell proliferation, as revealed by cell confluency.

c, Efficient knockdown of circFAM120A by shRNAs in HeLa cells. The expression of circRNAs and cognate linear RNAs was detected by qRT-PCR.

d, Knockdown of circFAM120A by shRNAs inhibited cell proliferation in HeLa cells, as revealed by MTT assay.

e, Efficient knockdown of circFAM120A by shRNAs in 293FT cells. The expression of circRNAs and cognate linear RNAs was detected by qRT-PCR.

f, Knockdown of circFAM120A by shRNA inhibited cell proliferation in 293FT cells, as revealed by MTT assay.

b-d,f, Values represent mean +/- SD with $n=3, *: P<0.05 ; * *: P<0.01$.

c-e, All transcript levels were normalized to $A C T B$, and values represent mean +/- SD with $n=3$. 
a

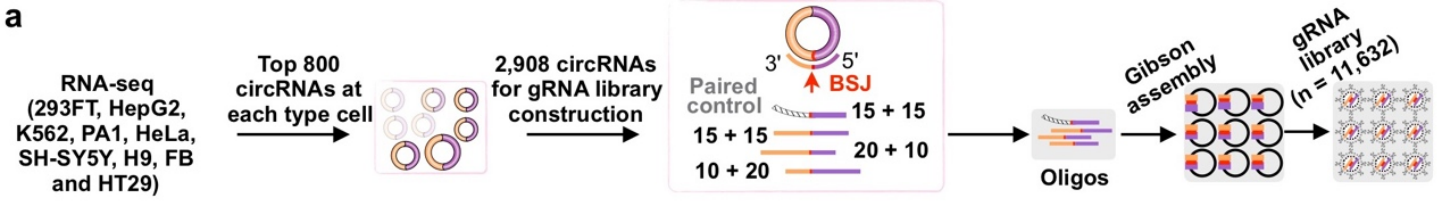

b

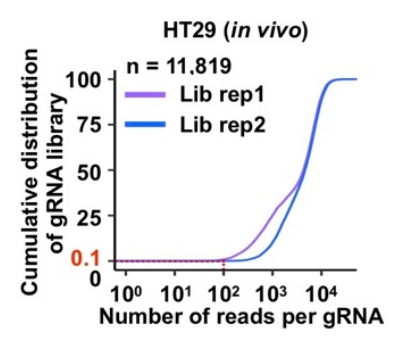

C

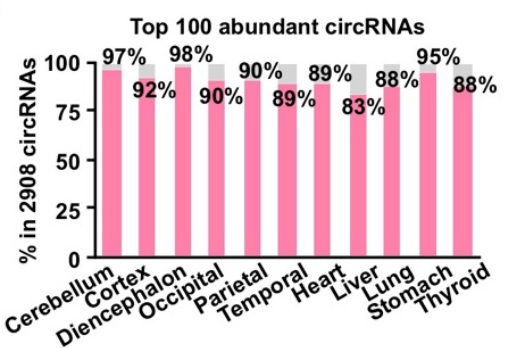

d

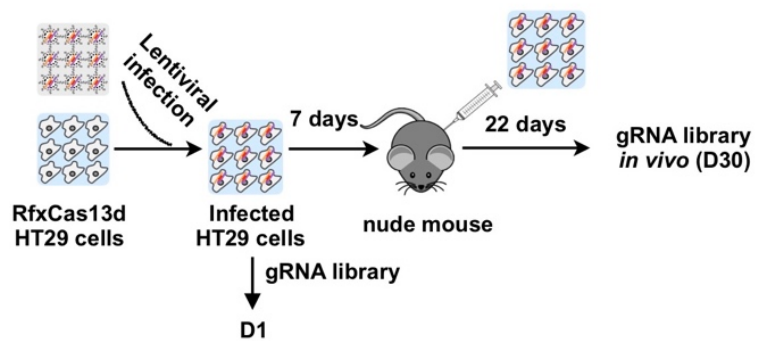

e

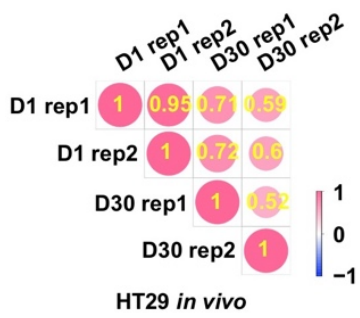

Extended Data Fig. 9 | Overview of sequencing analyses of in vivo screens using BSJ-gRNA libraries targeting 2,908 circRNAs.

a, Construction of an additional gRNA library targeting 2,908 circRNAs. One paired control gRNA $(n=2,908)$ and three BSJ-gRNAs (circRNA gRNAs, $n=8,724)$ were designed for each candidate circRNA. 100 negative control gRNAs were also included. b, Cumulative distribution of the number of reads per gRNA of constructed libraries.

The red line indicates that less than $0.1 \%$ of gRNAs are covered by less than 100 reads. c, Representation of 2,908 candidate circRNAs in the library in different human tissues $^{43}$. On average, over $90 \%$ of top 100 abundant circRNAs in each tissue were included in the list of 2,908 candidate circRNAs.

d, In vivo screen of circRNAs important for cell growth and proliferation. The gRNA lentiviral library was individually delivered into HT29 cells stably expressing RfxCas $13 \mathrm{~d}$. Infected cells were enriched after 7 days and injected subcutaneously to nude mouse for 22 days. Genomic DNAs from infected cells were extracted at day 1 (D1) and 30 (D30) for gRNA amplification and deep sequencing.

e, The Pearson correlation coefficient (PCC) between replicates (rep) of D1 and D30 in vivo samples in HT29. Two biologically independent experiments were performed at D1 and D30.

f, Scatter plot of fold change of paired controls and circRNA BSJ-gRNAs between D1 and D30 in vivo samples in HT29. The grey dashed lines indicate 2 or 0.5 fold change, respectively. 
a

\begin{tabular}{c|c|c|c}
\hline $\begin{array}{c}\text { Treatment } \\
(\text { poly(A)+) }\end{array}$ & Replicates & $\begin{array}{c}\text { \# of total } \\
\text { reads }\end{array}$ & $\begin{array}{c}\% \text { of mapped } \\
\text { reads }\end{array}$ \\
\hline \multirow{2}{*}{ RfxCas13d NT } & rep1 & $19,879,128$ & $83.6 \%$ \\
\cline { 2 - 4 } & rep2 & $23,224,224$ & $87.2 \%$ \\
\hline circFAM120A & rep1 & $20,730,415$ & $87.1 \%$ \\
\cline { 2 - 4 } gRNA1 (15nt + 15nt) & rep2 & $20,747,388$ & $88.0 \%$ \\
\hline \multirow{2}{*}{ shRNA NT } & rep1 & $21,071,165$ & $87.0 \%$ \\
\cline { 2 - 4 } & rep2 & $24,223,141$ & $86.4 \%$ \\
\hline \multirow{2}{*}{ circFAM120A } & rep1 & $21,583,441$ & $83.4 \%$ \\
\cline { 2 - 4 } shRNA1 (10nt + 11nt) & rep2 & $26,119,855$ & $89.0 \%$ \\
\hline & & &
\end{tabular}

c

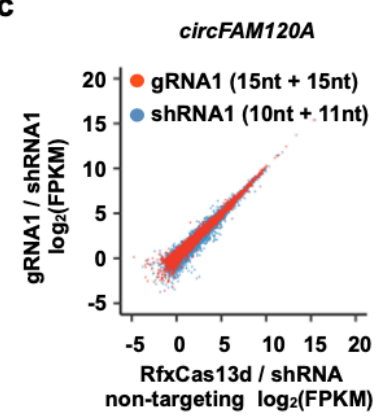

f

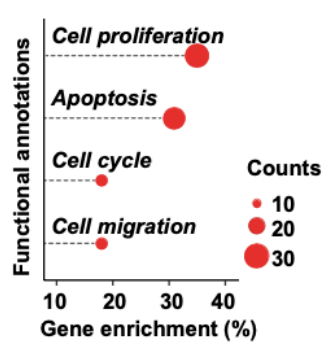

d

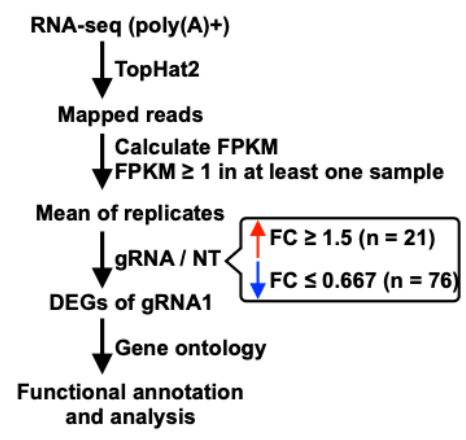

g

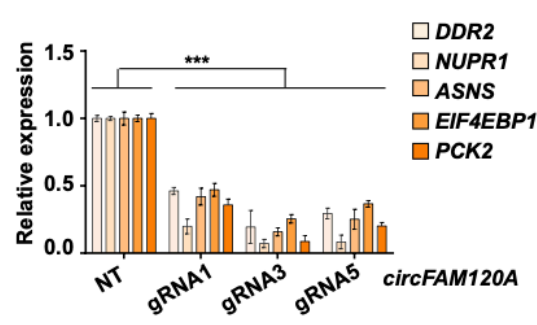

e b
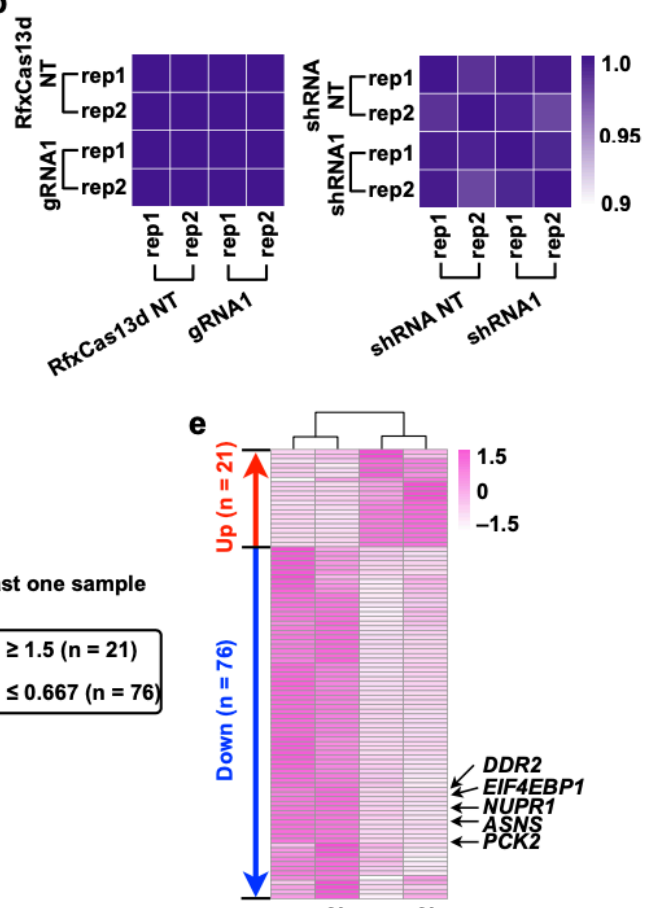

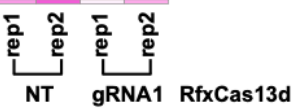

Extended Data Fig. 10 | RNA-seq analysis of RfxCas13d/BSJ-gRNA- or shRNAmediated $\operatorname{circFAM120A}$ knockdown.

a, Mapping statistics of two biological replicates of the poly(A)+ RNA-seq datasets in 293FT cells with RfxCas13d/BSJ-gRNA- or shRNA- mediated circFAM120A knockdown.

b, Heatmap of correlation (Kendall's tau) for $\log _{2}$ (FPKM) values of all linear mRNAs detected in RNA-seq libraries between targeting and non-targeting replicates for RfxCas13d/BSJ-gRNA- or shRNA- mediated circFAM120A knockdown.

c, The expression levels in $\log _{2}(\mathrm{FPKM})$ values of all genes detected in RNA-seq libraries of non-targeting control (x-axis) compared to circFAM120A-targeting conditions (y-axis) by RfxCas13d/BSJ-gRNA (red) or shRNA (blue). Means of two biological replicates were shown.

d, A workflow shows the selection of candidate genes after circFAM120A knockdown by RfxCas13d/BSJ-gRNA.

e, Heatmap of DEGs ( $\mathrm{n}=97)$ detected in 293FT cells after knocking down circFAM120A targeted by RfxCas13d/BSJ-gRNA.

f, Enrichment analysis of differentially expressed genes (DEGs) from RNA-seq after circFAM120A knockdown by RfxCas13d/BSJ-gRNA in 293FT cells. The $\mathrm{x}$ axis shows the ratio of the number of genes in a given category of functional annotations divided by the total number of DEGs. The y axis shows categories of functional annotations.

g, Validation of DEGs associated with cell proliferation after circFAM120A knockdown by $\mathrm{RfxCas} 13 \mathrm{~d} / \mathrm{BSJ}$-gRNA in $293 \mathrm{FT}$ cells. All transcript levels were normalized to $A C T B$, and values represent mean $+/-\mathrm{SD}$ with $n=3, * * *: P<0.001$. 
a

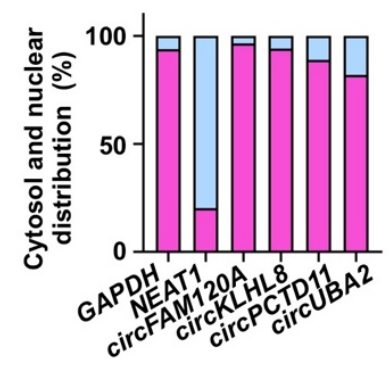

b

e

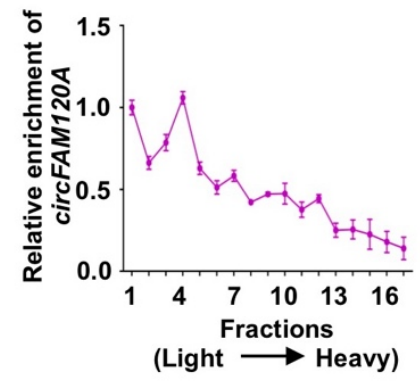

C

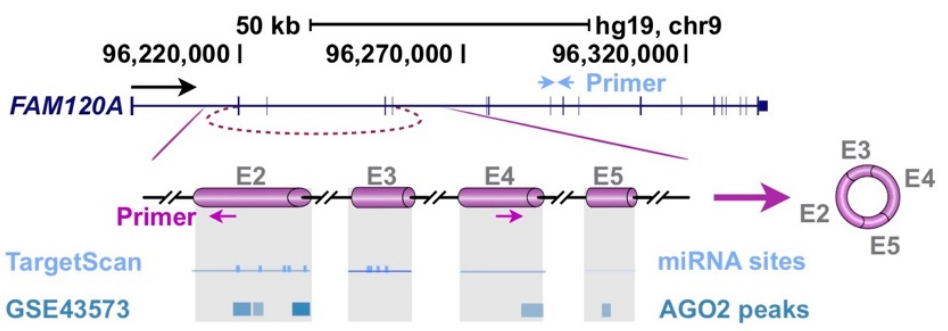

d

Extended Data Fig. 11 | CircFAM120A does not act as miRNA sponge.

a, Cytoplasmic distribution of circFAM120A. Total RNAs were separated into cytoplasmic and nuclear soluble fractions. The distribution of RNAs in the cytoplasmic and nuclear soluble fractions were detected by qRT-PCR.

b, Cytoplasmic extracts from 293FT cells were loaded on $10 \%-45 \%$ sucrose gradients. The enrichment of circFAM120A in individual fractions were measured by qRT-PCR. Fraction density increases from left to right.

c, Prediction of AGO2-binding peaks in circFAM120A. Top, genomics locus and diagram of linear FAM120A and circFAM120A (shown as cylinders in magenta). Blue and magenta arrows indicate location of primer for linear FAM120A or circFAM120A, respectively. Bottom, predicted miRNA target sites by TargetScan and AGO2 binding peaks from PAR-CLIP data in 293FT cells (GEO:GSE43573).

d, A schematic drawing to show the strategy for predication between circular RNA and miRNA. Sequences of circRNA and miRNA seeds were used to predicted circRNAmiRNA target sites by TargetScan. The predicted binding sites were validated with AGO2 PAR-CLIP dataset (GSE43573), and broadly conserved miRNAs were used for the subsequent analysis. After that, expressed miRNAs were selected for future analysis in 293FT cells (GSE58127/ERX350061).

e, CircFAM120A does not bind to AGO2 protein by RIP in 293FT cells using antiAGO2 antibodies. The percentage of RIP-enriched RNAs relative to input was quantified by qRT-PCR. 
a

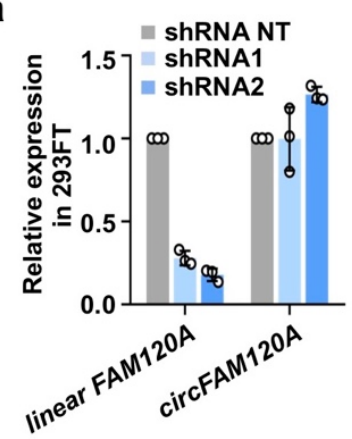

d

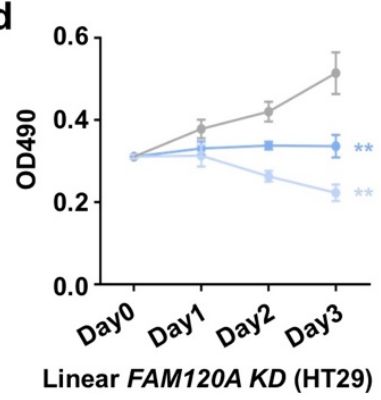

g

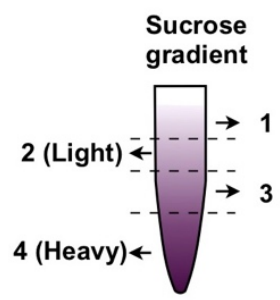

b

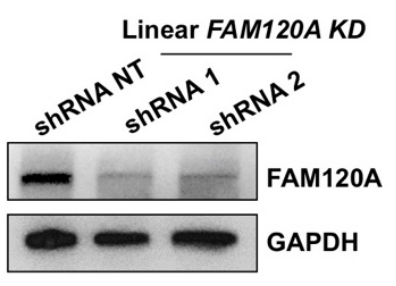

e
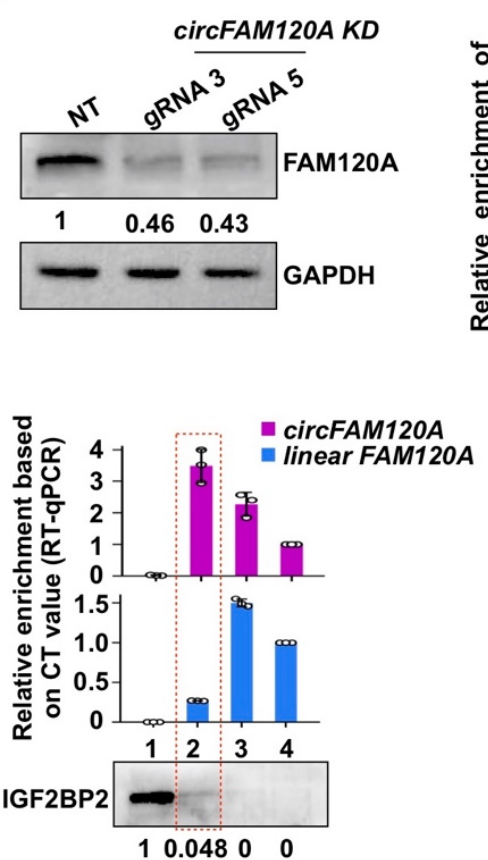

f c
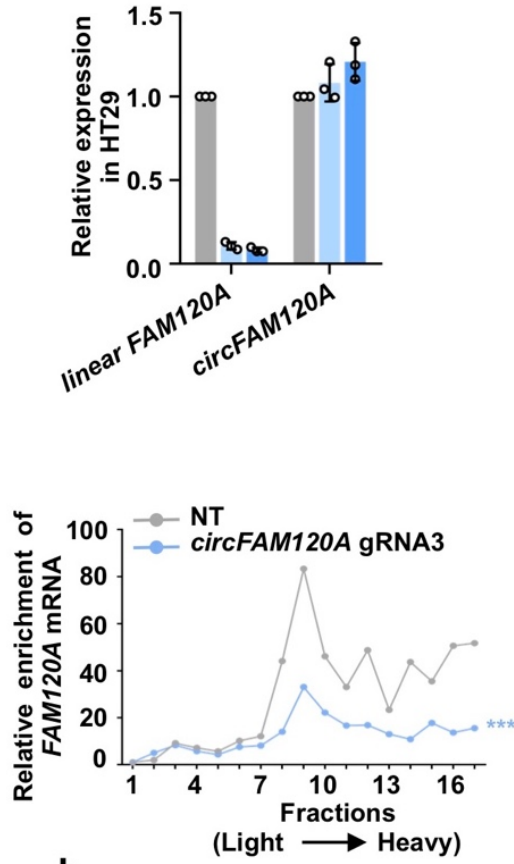

h

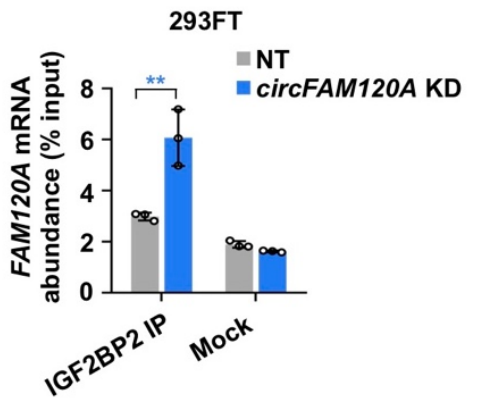

Extended Data Fig. 12 | CircFAM120A promotes cell proliferation by regulating its parental gene translation in an IGF2BP2-dependent manner.

a, Efficient knockdown of FAM120A by shRNAs in 293FT cells. The expression of circRNAs and cognate linear RNAs was detected by qRT-PCR, all transcript levels were normalized to $A C T B$, and values represent mean $+/$ - SD with $n=3$.

b, FAM120A knockdown by two different shRNAs in 293FT cells, revealed by WB.

c, Efficient knockdown of FAM120A by shRNAs in HT29 cells. The expression of circRNAs and cognate linear RNAs was detected by qRT-PCR.

d, Knockdown of FAM120A by shRNAs inhibited cell proliferation in HT29 cells, as revealed by MTT assay, and values represent mean $+/$ - SD with $n=3, * *: P<0.01$.

e, Knockdown of circFAM120A leads to reduced expression of FAM120A protein in HT29 cells.

f, Knockdown of circFAM120A leads to reduced enrichment of linear FAM120A on polyribosomes. Cytoplasmic extracts from 293FT cells treated with NT-gRNA (gray) or circFAM120A-gRNA3 (blue), were loaded on 10\%-45\% sucrose gradients. Enrichments of FAM120A mRNA in individual fractions were measured by qRT-PCR. Fraction density increases from left to right. Statistically significance was assessed using Wilcoxon test with R platform (R v.3.5.1) and values represent mean $+/$ - SD with $n=3, * * *: P<0.001$.

g, The distribution of IGF2BP2 protein, circFAM120 and FAM120A mRNA in four fractions of polysome profiling of 293FT cells. The schematic of the sucrose gradients 
used to segregate fractions in polysome profiling assay is shown on left.

h, Knockdown of circFAM120A leads to increased interaction between cognate FAM120A mRNA and IGF2BP2 in 293FT cells. The abundance of FAM120A mRNA was measured by qRT-PCR, and values represent mean $+/-\mathrm{SD}$ with $n=3, * *: P<0.01$.

a

b
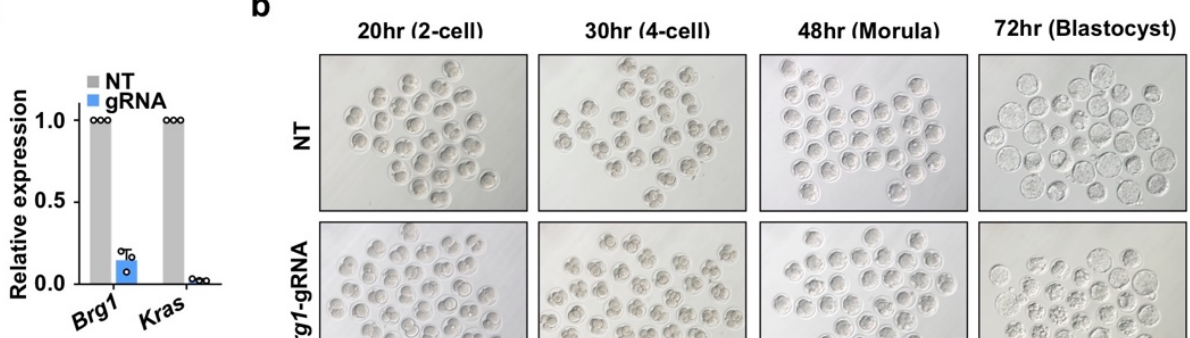

96hr (Expanded
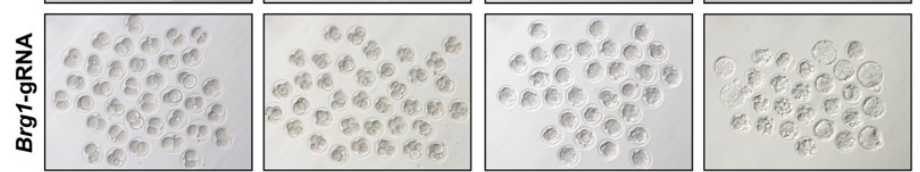
Blastocyst)

C
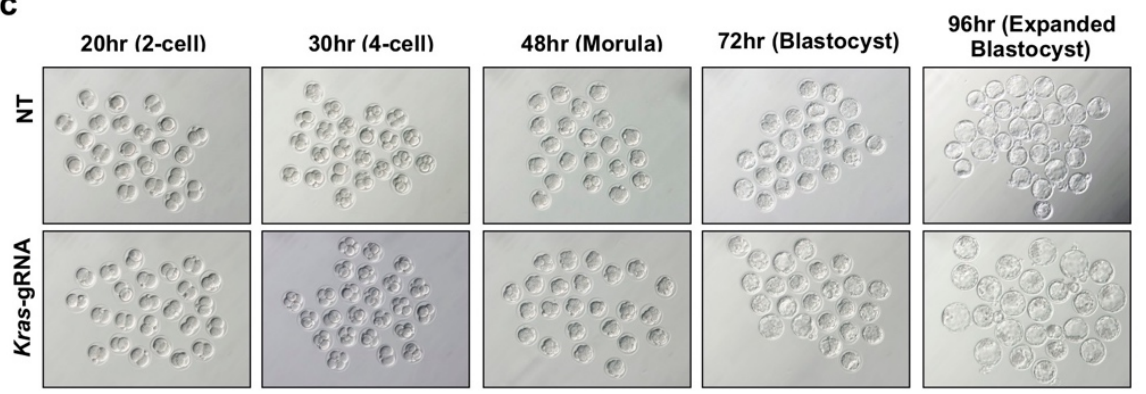

d

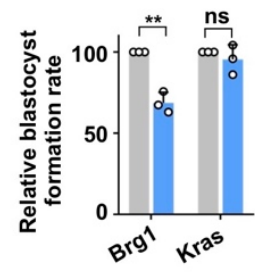

Extended Data Fig. 13 | Application of RfxCas13d/gRNA to interfere linear RNA expression during mouse preimplantation development.

a, Knockdown of Brgl and Kras by RfxCas13d/gRNA in zygotes. Expression levels of mRNAs were detected by qRT-PCR. All transcript levels are relative to $A C T B$, and values represent mean $+/$ - SD with $n=3$.

b, Representative images of reduced blastocyst formation $96 \mathrm{~h}$ after microinjection of RfxCas13d mRNA and the gRNA targeting Brgl mRNA into mouse zygotes. An example of failed blastocyst formation is shown by red arrows and enlarged view.

c, Representative images of embryogenesis after microinjection of RfxCas $13 \mathrm{~d}$ mRNA and the gRNA targeting Kras mRNA into mouse zygotes. Of note, no aberrant mouse preimplantation development was observed.

d, Effect of Brgl and Kras knockdown on blastocyst formation 96h after microinjection of RfxCas13d mRNA and BSJ-gRNAs into mouse zygotes. 
a

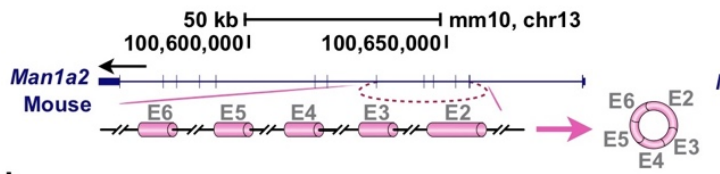

b
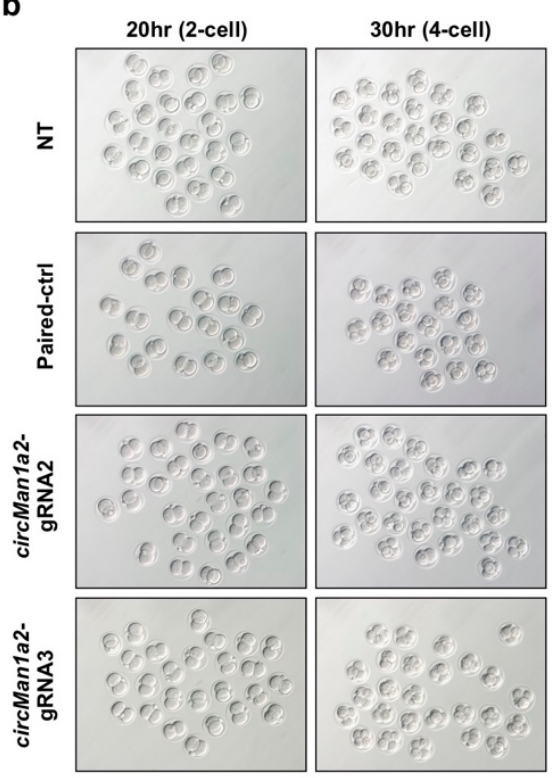

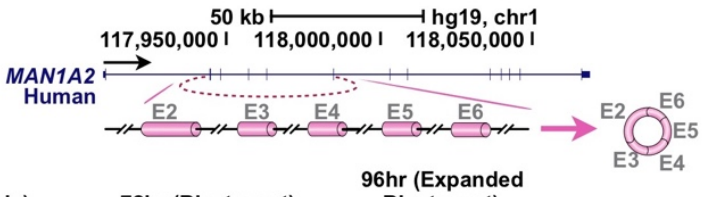

$48 \mathrm{hr}$ (Morula)

Blastocyst)
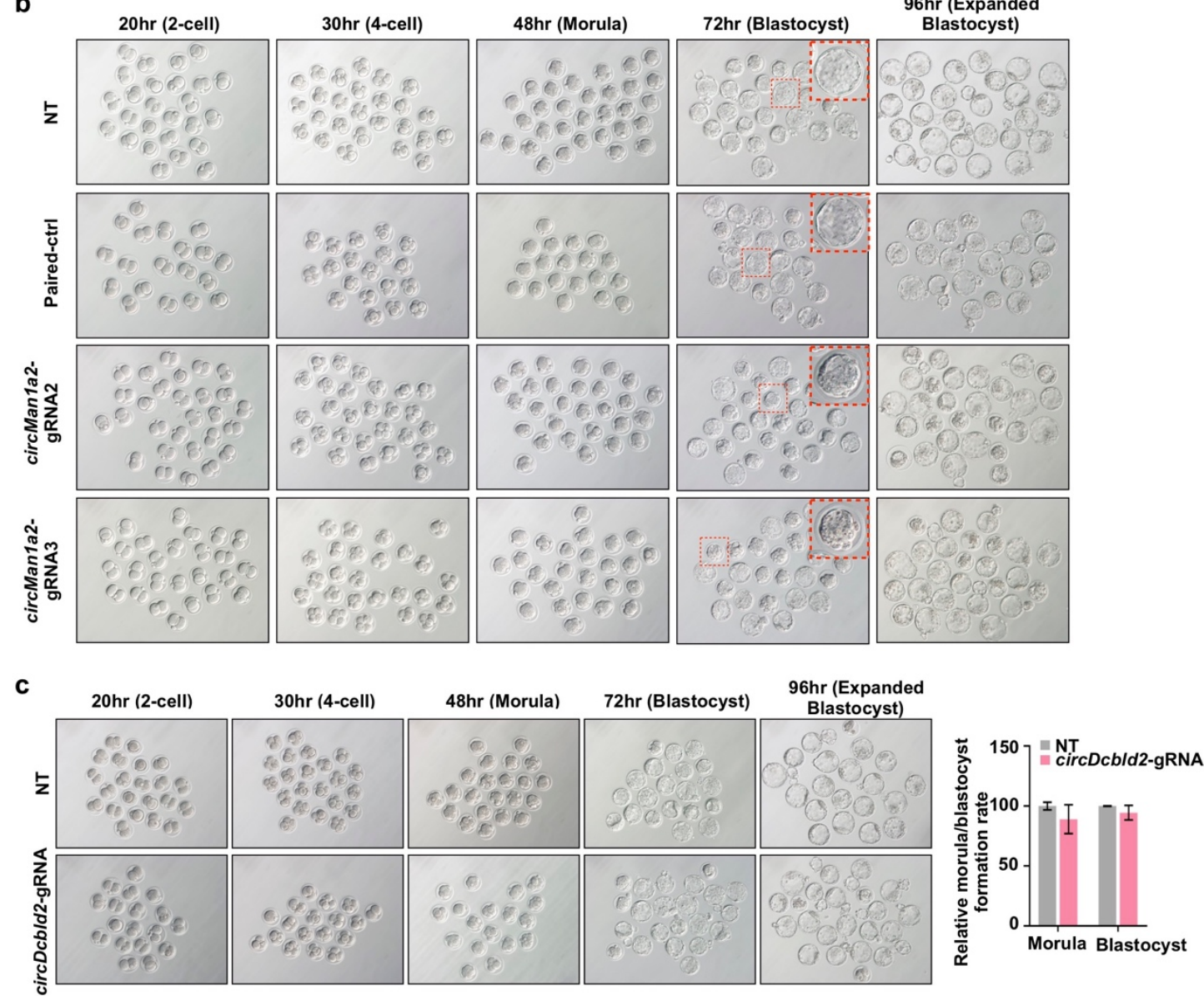

Extended Data Fig. 14 | Application of RfxCas13d/gRNA to interfere circRNA expression during mouse preimplantation development.

a, Genomic loci and diagrams of mouse circManla2 and human circMAN1A2 were shown as cylinders in magenta.

b, Knockdown of circMan $1 \mathrm{a} 2$ in zygotes led to reduced blastocyst formation $72 \mathrm{~h}$ after microinjection of RfxCas13d mRNA and the corresponding BSJ-gRNAs into mouse zygotes. Representative images of at 2-cell, 4-cell, morula and blastocyst stages are shown; an example under each condition is highlighted by red line and enlarged view. c, Images show the normal embryonic morphologies at 2-cell, 4-cell, morula and blastocyst stages under circDcbld2 knockdown by RfxCas13d/BSJ-gRNA in zygotes. The effect of $\operatorname{circDcbld} 2$ knockdown on morula and blastocyst formation was showed on right.

\section{References:}

38. Yin, Q. F. et al. Long noncoding RNAs with snoRNA ends. Mol. Cell 48, 219230 (2012).

39. Zhu, S. Y. et al. Genome-scale deletion screening of human long non-coding 
RNAs using a paired-guide RNA CRISPR-Cas9 library. Nat. Biotechnol. 34, 1279-1286 (2016).

40. Shalem, O. et al. Genome-scale CRISPR-Cas9 knockout screening in human cells. Science 343, 84-87 (2014).

41. Yang, L., Duff, M. O., Graveley, B. R., Carmichael, G. G. \& Chen, L. L. Genomewide characterization of non-polyadenylated RNAs. Genome Biol. 12, R16 (2011).

42. Wang, Y. et al. Genome-wide screening of NEAT1 regulators reveals crossregulation between paraspeckles and mitochondria. Nat. Cell Biol. 20, 1145-+ (2018).

43. Dong, R., Ma, X. K., Li, G. W. \& Yang, L. CIRCpedia v2: An Updated Database for Comprehensive Circular RNA Annotation and Expression Comparison. Genomics Proteomics Bioinformatics 16, 226-233 (2018).

\section{Supplementary Tables and Table Legends}

Supplementary Table 1. List of sequences of RfxCas13d/BSJ-gRNA library for 762 circRNAs in this study.

a, The expression (FPBcirc) of 762 circRNAs in HT29, 293FT and HeLa cells.

b, The sequences of RfxCas13d/BSJ-gRNA library for 762 circRNAs.

Supplementary Table 2. MAGeCK results of negatively selected circRNAs in HT29, 293FT and HeLa cell lines.

a, The robust rank aggregation (RRA) scores of ranked circRNAs in HT29 cells (D30/D1).

b, RRA scores of ranked circRNAs in 293FT cells (D30/D1).

c, RRA scores of ranked circRNAs in HeLa cells (D30/D1).

Supplementary Table 3. List of the normalized reads of top 50 candidate circRNAs identified by RfxCas13d/BSJ-gRNA screening in HT29, 293FT or HeLa cell lines.

a, Normalized reads of gRNAs for 50 candidate circRNAs in HT29 cells.

b, Normalized reads of gRNAs for 50 candidate circRNAs in 293FT cells.

c, Normalized reads of gRNAs for 50 candidate circRNAs in HeLa cells. 


\begin{tabular}{|c|c|c|c|c|c|c|c|}
\hline \multirow{2}{*}{$\begin{array}{l}\text { Parental } \\
\text { gene }\end{array}$} & \multirow{2}{*}{$\begin{array}{c}\text { CircRNA } \\
\text { (Chr:Start-End) } \\
\text { Conserved (mm10) }\end{array}$} & \multirow{2}{*}{$\begin{array}{c}\text { \# gRNAs with } \\
\text { KD efficiency }(>50 \%) \\
\text { /\# total validated gRNAs }\end{array}$} & \multirow{2}{*}{$\begin{array}{l}\text { Inhibition of } \\
\text { cell } \\
\text { proliferation }\end{array}$} & \multicolumn{3}{|c|}{ FPBcirc } & \multirow{2}{*}{$\begin{array}{l}\text { RRA rank } \\
\text { In vitro } L I\end{array}$} \\
\hline & & & & HT29 & 293FT & HeLa & \\
\hline$T J P 1$ & chr15:30053341-30092905 & 1/1 gRNA & $\checkmark$ & 0.41 & - & 0.71 & 6 \\
\hline NFATC3 & chr16:68155889-68157024 & 1/1 gRNA & $x$ & 1.53 & 1.58 & 0.78 & 10 \\
\hline DCBLD2 & chr3:98568304-98600611 & 1/1 gRNA & $\checkmark$ & 0.98 & 0.41 & - & 15 \\
\hline CSNK1G3 & chr5:122881110-122911657 & 1/1 gRNA & $\checkmark$ & 0.68 & 0.10 & 0.28 & 17 \\
\hline ERBB2IP & chr5:65284462-65290692 & 1/1 gRNA & $\checkmark$ & 2.15 & 0.33 & 1.91 & 20 \\
\hline FAM120A & chr9:96233422-96261168 & 3/3 gRNA & $\checkmark$ & 1.78 & 1.32 & 1.41 & 22 \\
\hline$A M D 1$ & chr6:111208707-111211559 & 1/1 gRNA & $\checkmark$ & 1.03 & 0.95 & 0.78 & 27 \\
\hline BPTF & chr17:65941524-65944422 & 1/1 gRNA & $\checkmark$ & 0.31 & 0.08 & 0.15 & 30 \\
\hline PCMTD1 & chr8:52773404-52773806 & 1/1 gRNA & $x$ & 2.67 & 2.12 & 5.28 & 48 \\
\hline$K L H L 8$ & chr4:88116475-88116842 & 3/3 gRNA & $\checkmark$ & 0.44 & 1.52 & 1.47 & 1 \\
\hline AMBRA1 & chr11:46515157-46529920 & 0/1 gRNA & $x$ & 0.28 & 0.36 & 0.37 & 4 \\
\hline FAM120A & chr9:96233422-96261168 & 3/3 gRNA & $\checkmark$ & 1.78 & 1.32 & 1.41 & 8 \\
\hline FAF1 & chr1:51253671-51267349 & 1/1 gRNA & $\checkmark$ & - & $1.60^{*}$ & 0.09 & 10 \\
\hline PMS1 & chr2:190656515-190682906 & 1/1 gRNA & $\checkmark$ & 1.12 & 1.04 & 1.78 & 12 \\
\hline ATXN2 & chr12:111990083-111993723 & 1/1 gRNA & $\checkmark$ & 2.21 & 0.65 & 3.13 & 16 \\
\hline UBAP2 & chr9:33971648-33973235 & 1/1 gRNA & $\checkmark$ & 0.20 & 4.66 & 0.44 & 17 \\
\hline PDCD11 & chr10:105197771-105198565 & 1/1 gRNA & $\checkmark$ & - & 0.17 & 0.09 & 21 \\
\hline EXOC6B & chr2:72945231-72960247 & 1/1 gRNA & $\checkmark$ & 0.73 & 1.66 & 2.60 & 28 \\
\hline CCNB1 & chr5:68470703-68471364 & 1/1 gRNA & $\checkmark$ & 0.99 & 1.47 & 1.62 & 37 \\
\hline CLASP1 & chr2:122363276-122363756 & 1/1 gRNA & $\checkmark$ & 0.42 & 0.57 & 0.46 & 9 \\
\hline FAM120A & chr9:96233422-96261168 & $3 / 3$ gRNA & $\checkmark$ & 1.78 & 1.32 & 1.41 & 12 \\
\hline XPO1 & chr2:61749745-61761038 & 1/1 gRNA & $\checkmark$ & 4.69 & 4.52 & 3.65 & 13 \\
\hline$K L H L 8$ & chr4:88116475-88116842 & 3/3 gRNA & $\checkmark$ & 0.44 & 1.52 & 1.47 & 20 \\
\hline ATXN10 & chr22:46085591-46098727 & 0/1 gRNA & $x$ & 0.31 & 0.44 & 0.79 & 22 \\
\hline$A S P H$ & chr8:62593526-62596747 & 1/1 gRNA & $\checkmark$ & 4.88 & 1.95 & 11.93 & 29 \\
\hline HIPK3 & chr11:33307958-33309057 & $3 / 3$ gRNA & $\checkmark$ & 10.92 & 6.23 & 15.97 & 35 \\
\hline MIB1 & chr18:19345732-19359646 & 1/1 gRNA & $\checkmark$ & 1.75 & 7.26 & 3.18 & 36 \\
\hline CRKL & chr22:21288066-21288532 & 1/1 gRNA & $\checkmark$ & 3.25 & 4.93 & 1.20 & 46 \\
\hline
\end{tabular}

Supplementary Table 4. List of validated circRNA candidates by $R f x C a s 13 d / B S J-$ gRNA in HT29, HeLa and 293FT cells.

Their parental genes, genomic locations, FPBcirc, RRA ranks are listed. Most of examined candidate circRNAs can be validated by RfxCas13d with at least one gRNA in HT29, HeLa and 293FT cells, respectively.

Supplementary Table 5. List of sequences of RfxCas13d/BSJ-gRNA library for 2908 circRNAs in this study.

a, The sequences of RfxCas13d/BSJ-gRNA library for 2908 circRNAs.

b, The robust rank aggregation (RRA) scores of ranked circRNAs in HT29 in vivo (D30/D1).

c, The top 200 robust rank aggregation (RRA) scores of ranked circRNAs in HT29 in vivo (D30/D1).

d, Overlap of top 50 in vitro and top 200 in vivo robust rank aggregation (RRA) scores of ranked circRNAs in HT29 (D30/D1). 
bioRxiv preprint doi: https://doi.org/10.1101/2020.03.23.002865; this version posted March 25, 2020. The copyright holder for this preprint (which was not certified by peer review) is the author/funder, who has granted bioRxiv a license to display the preprint in perpetuity. It is made available under aCC-BY-NC-ND 4.0 International license.

\begin{tabular}{|c|c|c|c|c|c|c|c|c|c|c|c|c|c|c|}
\hline \multicolumn{2}{|c|}{ Mouse $(\mathrm{mm} 10)$} & \multicolumn{7}{|c|}{ Mouse (FPBcirc) } & \multirow{2}{*}{$\begin{array}{c}\begin{array}{c}\text { Mouse } \\
\text { (FPBcirc) }\end{array} \\
\text { R1 }\end{array}$} & \multicolumn{5}{|c|}{ Relative expression to $A c t b$} \\
\hline $\begin{array}{l}\text { CircRNA genomic location } \\
\text { (Chr:Start-End) }\end{array}$ & Parental gene & oocyte & zygote & 2cell & 4cell & 8cell & morula & blastocyst & & zygote & 2cell & 4cell & morula : & blastocyst \\
\hline chr7:65336031-65354888 & Tjp1 & 0.00 & 0.00 & 1.08 & 0.00 & 0.00 & 0.00 & 0.00 & - & 3.46 & 1.47 & 2.01 & 0.36 & 0.03 \\
\hline chr18:53895530-53919033 & Csnk1g3 & 0.00 & 0.18 & 0.00 & 0.00 & 0.00 & 0.22 & 0.00 & - & 0.00 & 0.03 & 0.20 & 0.02 & 0.00 \\
\hline chr16:58424560-58433463 & Dcbld2 & - & - & - & - & - & - & - & 0.58 & 0.04 & 0.15 & 0.21 & 0.01 & 0.00 \\
\hline chr1:7120193-7120615 & Pcmtd1 & - & - & - & - & - & - & - & 0.19 & 0.00 & 0.01 & 0.02 & 0.00 & 0.00 \\
\hline chr2:104470748-104471847 & Hipk3 & 0.59 & 0.09 & 0.00 & 0.00 & 0.00 & 0.00 & 0.00 & 0.39 & 0.04 & 0.23 & 0.35 & 0.05 & 0.00 \\
\hline chr11:23261834-23271205 & Xpo1 & 0.00 & 0.00 & 0.72 & 0.00 & 0.00 & 0.00 & 0.00 & 0.19 & 0.00 & 0.00 & 0.04 & 0.00 & 0.00 \\
\hline chr4:9635900-9639347 & Asph & 0.00 & 0.00 & 3.41 & 0.00 & 0.00 & 1.90 & 0.00 & 10.41 & 0.17 & 0.84 & 1.07 & 0.06 & 0.00 \\
\hline chr1:118419439-118419918 & Clasp1 & - & - & - & - & - & - & - & 1.35 & 0.00 & 0.01 & 0.05 & 0.00 & 0.00 \\
\hline chr15:85359453-85376702 & Atxn10 & - & - & - & - & - & - & - & 0.77 & 0.00 & 0.00 & 0.01 & 0.00 & 0.00 \\
\hline chr5:103885906-103886290 & KIh/8 & - & - & - & - & - & - & - & 0.39 & 0.05 & 0.18 & 0.23 & 0.01 & 0.00 \\
\hline chr1:53256628-53282092 & Pms1 & 0.68 & 0.18 & 1.79 & 0.00 & 0.00 & 0.00 & 0.00 & 0.19 & 0.19 & 0.73 & 0.95 & 0.06 & 0.00 \\
\hline chr2:91810029-91825352 & Ambra1 & - & - & - & - & - & - & - & 2.51 & 0.01 & 0.06 & 0.16 & 0.01 & 0.00 \\
\hline chr5:121745722-121749229 & Atxn2 & - & - & - & - & - & - & - & 2.31 & 0.01 & 0.03 & 0.10 & 0.01 & 0.00 \\
\hline chr6:84989324-85005036 & Exoc6b & - & - & - & - & - & - & - & 0.29 & 0.00 & 0.01 & 0.02 & 0.00 & 0.00 \\
\hline chr13:48932296-48949303 & Fam120a & - & - & - & - & - & - & - & 0.19 & 0.02 & 0.07 & 0.16 & 0.01 & 0.00 \\
\hline chrX:93461368-93493581 & Pola1 & 31.45 & 41.94 & 58.30 & 61.41 & 69.56 & 30.79 & 0.00 & 0.34 & 2.22 & 7.22 & 10.18 & 0.52 & 0.00 \\
\hline chr14:86810335-86918864 & Diaph3 & 6.83 & 12.38 & 54.89 & 51.62 & 41.17 & 24.10 & 0.76 & - & 0.15 & 0.64 & 1.02 & 0.06 & 0.00 \\
\hline chr10:25267307-25289730 & Akap7 & 14.33 & 11.84 & 24.40 & 39.15 & 33.87 & 28.00 & 0.13 & 0.34 & 0.17 & 0.70 & 0.97 & 0.06 & 0.00 \\
\hline chr3:100632503-100656274 & Man1a2 & 1.01 & 7.66 & 7.53 & 10.10 & 18.05 & 7.70 & 0.00 & 0.05 & 0.04 & 0.14 & 0.21 & 0.02 & 0.00 \\
\hline chr4:84971130-85006524 & Cntln & 5.73 & 4.99 & 22.24 & 36.94 & 46.64 & 10.60 & 0.00 & - & 0.13 & 0.62 & 0.85 & 0.06 & 0.00 \\
\hline chr9:22643744-22679064 & Bbs9 & 7.84 & 4.36 & 10.94 & 5.05 & 8.92 & 8.03 & 1.27 & 0.44 & 3.09 & 12.21 & 20.48 & 1.23 & 0.01 \\
\hline chrX:93420566-93493581 & Pola1 & 4.38 & 4.01 & 6.10 & 3.16 & 3.25 & 2.01 & 0.00 & - & 0.18 & 0.72 & 0.99 & 0.06 & 0.00 \\
\hline chr14:86810335-86835101 & Diaph3 & 1.52 & 4.01 & 5.56 & 9.79 & 2.64 & 9.60 & 0.00 & 0.08 & 1.26 & 4.07 & 7.49 & 0.40 & 0.00 \\
\hline chrX:93474312-93493581 & Pola1 & 1.94 & 3.92 & 3.59 & 9.00 & 0.00 & 0.56 & 0.00 & - & 0.18 & 0.74 & 0.97 & 0.05 & 0.00 \\
\hline
\end{tabular}

Supplementary Table 6. Expression of 24 circRNA candidates in mouse preimplantation development. 


\begin{tabular}{|c|c|c|c|c|}
\hline \multirow{2}{*}{ Parental gene } & \multirow{2}{*}{$\begin{array}{c}\text { CircRNA } \\
\text { (Chr:Start-End) }\end{array}$} & \multicolumn{3}{|c|}{$\%$ of mouse embryo preimplantation } \\
\hline & & 4-cell & Morula & Blastocyst \\
\hline Tjp1 & chr7:65336031:65354888 & 1.04 & 1.06 & 1.06 \\
\hline Csnk1g3 & chr18:53895530:53919033 & 1.01 & 0.86 & 1.00 \\
\hline Dcbld2 & chr16:58424560:58433463 & 0.97 & 0.97 & 0.99 \\
\hline Pcmtd1 & chr1:7120193:7120615 & 0.93 & 0.97 & 0.97 \\
\hline Fam120a & chr13:48932296:48949303 & 0.97 & 0.85 & 0.86 \\
\hline Hipk3 & chr2:104470748:104471847 & 0.97 & 0.97 & 0.96 \\
\hline Xpo1 & chr11:23261834:23271205 & 0.99 & 0.92 & 0.96 \\
\hline Asph & chr4:9635900:9639347 & 1.06 & 0.97 & 1.11 \\
\hline Clasp1 & chr1:118419439:118419918 & 1.10 & 1.14 & 1.14 \\
\hline Atxn10 & chr15:85359453:85376702 & 1.00 & 0.93 & 0.93 \\
\hline$K / h / 8$ & chr5:103885906:103886290 & 0.95 & 0.91 & 1.09 \\
\hline Pms1 & chr1:53256628:53282092 & 1.02 & 0.88 & 1.03 \\
\hline Ambra1 & chr2:91810029:91825352 & 1.03 & 0.98 & 1.07 \\
\hline Atxn2 & chr5:121745722:121749229 & 1.10 & 1.23 & 1.2 \\
\hline Exoc6b & chr6:84989324:85005036 & 1.09 & 0.92 & 1.06 \\
\hline Pola1-1 & chrX:93461368:93493581 & 0.99 & 0.86 & 0.89 \\
\hline Pola1-2 & chrX:93420566:93493581 & 0.94 & 1.00 & 0.92 \\
\hline Pola1-3 & chrX:93474312:93493581 & 0.91 & 0.90 & 0.89 \\
\hline Diaph3-1 & chr14:86810335:86918864 & 0.94 & 0.84 & 0.86 \\
\hline Diaph3-2 & chr14:86810335:86835101 & 1.00 & 0.91 & 0.98 \\
\hline Akap7 & chr10:25267307:25289730 & 1.00 & 0.81 & 1.03 \\
\hline Man1a2 & chr3:100632503:100656274 & 0.98 & 0.85 & 0.65 \\
\hline Cnt/n & chr4:84971130:85006524 & 1.16 & 0.94 & 0.94 \\
\hline Bbs9 & chr9:22643744:22679064 & 1.00 & 0.80 & 0.97 \\
\hline
\end{tabular}

Supplementary Table 7. Screening of circRNAs with potential regulatory roles in mouse embryo preimplantation development by RfxCas13d/BSJ-gRNA KD each of 24 circRNA candidates in mouse zygotes.

Supplementary Table 8. Expression of linear genes upon $\operatorname{circFAM120A}$ knockdown by RfxCas13d or shRNA in 293FT cells.

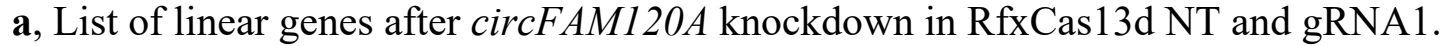

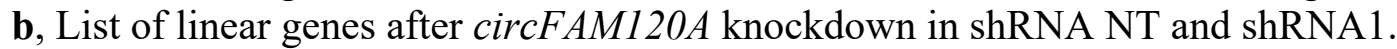

Supplementary Table 9. List of sequences used in this study.

a, gRNA sequences.

b, shRNA sequences.

c, Primers for qRT-PCR and NB probes. 